Dieses Dokument ist eine Zweitveröffentlichung (Verlagsversion) / This is a self-archiving document (published version):

Alexander Lasch

Das Fenster wirkt geschlossen - Überlegungen zu nonagentiven Konstruktionen des Deutschen aus konstruktionsgrammatischer Perspektive

Erstveröffentlichung in / First published in:

Alexander Lasch/Alexander Ziem, Hrsg. Grammatik als Netzwerk von Konstruktionen. Berlin: De Gruyter 2014, S. 65 - 93. ISBN 978-3-11-035369-3.

DOI: https://doi.org/10.1515/9783110353693.65

Diese Version ist verfügbar / This version is available on:

https://nbn-resolving.org/urn:nbn:de:bsz:14-qucosa2-748480 


\title{
Das Fenster wirkt geschlossen - Überlegungen zu nonagentiven Konstruktionen des Deutschen aus konstruktionsgrammatischer Perspektive
}

\begin{abstract}
Die in diesem Beitrag angestellten Überlegungen sind Teil breiter angelegter Studien zu nonagentiven Konstruktionen des Deutschen, die im Hinblick auf ihre Perspektivierungsleistung modelliert werden. Ziel ist, das „Konversen'-Modell zur Erklärung passivischer und passivähnlicher Strukturen zu suspendieren. ${ }^{1}$ Mit anderen Worten: Die verbale Kategorie des Genus verbi wird zur Disposition gestellt und stattdessen ein Bereich des Konstruktikons mit einer Reihe miteinander vernetzter Konstruktionen mit spezifischer Perspektivierungsleistung postuliert. ${ }^{2}$ In diesem Artikel wird unter dieser übergreifenden Zielsetzung und auf der Basis einer qualitativen Untersuchung der Versuch unternommen, einen Vererbungsprozess zwischen Konstruktionen aufzuzeigen und einen Ausschnitt des Konstruktionsnetzwerks im Bereich der nonagentiven Konstruktionen des Deutschen zu beschreiben. ${ }^{3}$ Dabei wird das Verb wirken im Mittelpunkt stehen. Die Konstruktionen, in die das Verb wirken $u$. a. eingebettet sein kann, werden dabei als Typen der Konstruktion der Askription, der Eigenschaftszuweisung, aufgefasst, die typischerweise mit dem Verb sein gebildet werden, aber z. B. auch bleiben, erscheinen, scheinen $\mathrm{u}$. a. einbetten können.
\end{abstract}

\section{Hinführung}

Kernanliegen der Konstruktionsgrammatik ist es, Konstruktionen als ein(ziges) Format sprachlichen Wissens aufzufassen und so Spracherwerb, Sprachgebrauch und Sprachwandel adäquat zu beschreiben. ${ }^{4}$ Das schließt nicht nur die Analyse von (monolithischen) Konstruktionen ein, sondern zwingt dazu, sich Gedanken darüber zu machen, wie Kon-

1 Dazu zählen die Entwicklung eines Strukturmodells von Konstruktionen im Hinblick auf die Modellierung eines Konstruktikons auf der Basis von Goldberg (1995, 2006), Croft (2001) und von Polenz (2008) sowie die Diskussion etwa des Beispiels Leonard abgeholt! in Ziem \& Lasch (2013: 110-142 sowie 165-172, besonders 168f.).

2 Es spricht viel dafür, die von Eisenberg in Anlehnung an Anderson und Thieroff gezeichnete Gliederung der verbalen Kategorisierungen bis auf die relationale Kategorie (Genus verbi) zu übernehmen. Zu unterscheiden sind danach Kongruenzkategorien (Person und Numerus) sowie inhärente Kategorien (Modus und Tempus) (vgl. Eisenberg 2006 I: 205 und 2011). Die Anschlussdiskussion, wie mit den analytisch gebildeten Tempora angesichts der hier vorgeschlagenen Neubewertung in Zukunft umzugehen sei (diese werden auch von Eisenberg 2011 problematisiert), kann hier (noch) nicht geführt werden.

3 Vgl. dazu die Beiträge von Ziem und Boas (in diesem Band).

4 Vgl. einführend bspw. Goldberg (2003), Croft \& Cruse (2004) und Stefanowitsch (2011: 181-188). 
struktionen miteinander in Verbindung stehen, welche Vererbungsprozesse und Linkings zwischen Konstruktionen unterschiedlichen Abstraktionsgrads bestehen und wie man mittels dieses Netzwerks (Konstruktikon) ${ }^{5}$ komplexes sprachliches Wissen in seiner Strukturierung zu beschreiben in der Lage ist. ${ }^{6}$ Die Konstruktionsgrammatik steht außerdem nach Jahren der Diskussion theoretischer Prämissen und zahlreichen Erweiterungen sowie Anschlussversuchen an andere Grammatik- und Semantikmodelle in der Pflicht, konkrete Vorschläge zur Analyse sprachlicher Einheiten vor allem auch auf syntaktischer Ebene zu unterbreiten.

Nach einer knappen Skizze der Beschreibung des Verbs wirken in anderen funktionalen Grammatiken (Abschnitt 1) wird kurz einleitend die theoretische Basis des Beitrags erläutert (Abschnitt 2). Anschließend wird diskutiert, welche Gebrauchsvarianten von wirken in nonagentive Konstruktionen der Askription eingebettet werden können (Abschnitt 3). Der nächste Schritt ist der Korpusanalyse gewidmet, die wichtige Hinweise auf z. B. die Art der sprachlichen Einheiten liefert, die gemeinsam mit wirken auftreten (Abschnitt 4). Die Konstruktionen mit wirken werden schließlich im Konstruktionsnetzwerk verortet (Abschnitt 5). Abschließend werden die Ergebnisse zusammengefasst und weiterführende Fragen formuliert (Abschnitt 6).

\section{Das Fenster wirkt geschlossen - eine Standortbestimmung}

In aktuellen Grammatiken, etwa der Duden-Grammatik (vgl. 2009: 415), sind sein, werden und bleiben sowie scheinen, sich dünken und beißen Kopulaverben; Eisenberg rechnet nur die ersten drei zweifelsfrei dazu, scheinen nimmt er explizit aus. ${ }^{7}$ Das grammatische Informationssystem des Instituts für deutsche Sprache (IdS) Mannheim „grammis 2.0“ nennt daneben noch „kopulaähnliche Verben"8 wie gelten, nennen und aussehen; Eisenberg weiter sich dünken, klingen und schmecken, da sie ein ,adjektivisches Prädikatsnomen nehmen“, und heißen, das wie sich dïnken mit einem substantivischen Prädikatsnomen stehen kann (Eisenberg 2006 II: 86). Sie werden alle den so genannten Prädikativverben zugerechnet, also Verben, die „sich mit einem Subjekts- oder Objektsprädikativ zu einem mehrteiligen Prädikat“ verbinden. Das kann z. B. ein „prädikativer Nominativ“ sein, ein „,verkürzter Ausdruck für ,prädikative Nominalphrase im Nominativ““ (Duden-Grammatik 2009: 812):

(1) a. Das Modell ist/wird/bleibt ein Erfolg.

b. Dieser Entscheid scheint ein gewaltiger Fortschritt.

c. Der Entscheid dünkt mich ein gewaltiger Fortschritt.

d. Dieses Sternbild beißt Großer Bär. (Duden-Grammatik 2009: 813)

5 Die Präzisierung des Begriffs in diesem Sinne erfolgte durch Goldberg (1995: 5), vgl. ausführlicher auch Boas (2010) und Broccias (2012), um nur einige exemplarische Studien zu nennen. Ausführlich dazu Ziem einleitend (in diesem Band).

6 So u. a. Goldberg (2003: 219) sowie Stefanowitsch \& Gries (2003: 212) in dem für die Konstruktionsgrammatik einschlägigen Aufsatz zu so genannten Kollostruktionen, also kollokativ verbundenen Clustern und n-Grammen sprachlicher Einheiten mit Konstruktionsstatus.

7 Das allerdings greift zu kurz: Eisenberg lässt scheinen nur in Verbindung mit dem Infinitiv mit $2 u$ zu (vgl. Eisenberg 2006 II: 85f.; zu scheinen dann 353-365, besonders 362-365).

8 Artikel „Kopulaverb“ bei grammis 2.0; abrufbar unter: <http://goo.gl/3D43D >, Stand: 04.03.2014. 
Allen diesen Realisierungen ist gemeinsam, dass sie eine Nominalphrase im Nominativ in Bezug setzen zu einer anderen Nominalphrase im Nominativ, deren Minimalform die in (2) ist:

(2) a. Lewin ist/wird/bleibt Lehrer.

b. Lewin ?scheint/?dünkt mich Lebrer.

c. Lewin scheint Lebrer zu sein/zu werden/zu bleiben.

d. Lewin heißt Lebrer.

Die Varianten (2b) dürften tendenziell als unüblich gelten. Grund dafür ist, dass scheinen zusammen mit einem $z u$-Infinitiv eines Kopula auftreten kann wie in (2c) (Eisenberg 2006 II postuliert das gar als einzige Verwendung) und sich dïnken hinsichtlich seiner Frequenz im Sprachgebrauch deutlich zurückgeht. Anders verhält es sich in (2d): Die Aussage hat in vielen Gebrauchszusammenhängen schon eine gänzlich andere Bedeutung als (2a) und (2c). Hier wird (mittlerweile) bspw. nicht mehr auf eine Tätigkeit von Lewin abgehoben (,Man sagt, er sei Lehrer'), sondern auf seinen Eigennamen: ,Lewin trägt den Namen Lewin Lehrer. Daneben sind andere Restriktionen auszumachen, die hier nicht diskutiert werden sollen. ${ }^{9}$ Deutlich wird an diesen Beispielen, dass es sich bei Kopulaverben und kopulaähnlichen Verben um eine durch formale und semantische Merkmale bestimmte (offene) Klasse an Verben handelt. Die meisten dieser Verben können nicht nur Nominalphrasen im Nominativ verbinden (was ihr Name von lat. copulare bedeutet), sondern auch Nominalphrasen und (deverbale) Adjektive, wie (3) zeigt.

(3) a. Das Fenster ist/wird/ bleibt verschlossen.

b. Das Fenster scheint verschlossen.

Allen Varianten in (3) ist ein semantisches Merkmal gemeinsam: Sie thematisieren eine Eigenschaft des Fensters: In (3a) wird eine Eigenschaft betont, die das Fenster hat, haben wird oder behält. In (3b) wird eine (gegenüber der Kopula sein markierte) Faktizitätseinschätzung durch den Sprecher über eine Eigenschaft, die das Fenster hat, vorgenommen. Es sind Konstruktionen der Eigenschaftszuweisung, der Askription (vgl. Abschnitt 3). Die Forschung hat sich in den letzten Jahren vor allem auf die Varianten mit sein konzentriert; hier wurde die starre Bestimmung im Zusammenhang des so genannten Zustandspassivs aufgehoben zu Gunsten der Annahme eines Resultativums, das berücksichtigt, dass seinPerfekt, sein-Passiv sowie der oben erläuterte Verwendungszusammenhang als Kopulaverb enger zusammenstehen, als in der älteren Grammatikschreibung betont wurde. ${ }^{10}$

Im Folgenden wird dieser Zusammenhang im Mittelpunkt stehen, allerdings soll nicht die Konstruktion der Eigenschaftszuweisung mit sein besprochen werden, da hier der Raum für eine umfangreiche Forschungsdiskussion nicht gegeben ist. Stattdessen widme ich mich einem Verb, das als kopulaähnliches Verb zu verstehen ist und wie sein, werden, bleiben und scheinen in Konstruktionen der Eigenschaftszuweisung eingebettet ist zusammen mit (deverbalen) Adjektiven, wie in (4):

(4) Das Fenster wirkt verschlossen.

9 Vgl. dazu z. B. Eisenberg (2006 II: 85-90, bis auf scheinen).

10 Vgl. dazu exemplarisch Maienborn (2003), Welke (2005: 212-229), Boas (in diesem Band) und Diedrichsen (in diesem Band). 
Die Diskussion um wirken als „kopulaähnliches Verb“ ist, um es neutral zu sagen, noch keineswegs erschöpft: Die Duden-Grammatik nennt es bisher nicht; Gleiches gilt für das grammatische Informationssystem des IdS. Wirken in der hier andiskutierten Verwendung wird man dort nur in der Datenbank E-Valbu finden, dem elektronischen Valenzwörterbuch des IdS. Dort wird als fünfte Bedeutungsvariante des Verbs geführt: „,den Eindruck machen, als wäre es so/ein solches“. 11 Weiter wird man erfahren, dass „kein Passiv möglich" sei. Das verwundert nicht: Wirken in der Lesart, den Eindruck machen" (nach traditioneller Terminologie) leistet wie in (4) eingebettet in die Konstruktion der Askription selbst die Perspektivierung passivähnlicher Strukturen. Die Lesart wird unter bestimmten Bedingungen dabei durch die Konstruktion erzwungen (vgl. Goldberg 1995: 159f.; Michaelis 2003), wie im Folgenden zu zeigen sein wird.

\section{Gebrauchsbasierte konstruktionsgrammatische Ansätze}

Gebrauchsbasierte Ansätze beruhen in der Regel heute auf der Goldberg'schen Definition der Konstruktion: ${ }^{12}$

„Any linguistic pattern is recognized as a construction as long as some aspect of its form or function is not strictly predictable from its component parts or from other constructions recognized to exist. In addition, patterns are stored as constructions even if they are fully predictable as long as they occur with sufficient frequency.“ (Goldberg 2006: 5)

Im Gegensatz zu Goldbergs früherer Definition (Goldberg 1995: 5) wird hier deutlich herausgestellt, dass auch sprachliche Einheiten, die vollständig vorhersagbar sind, als Konstruktionen gelten sollen, solange sie nur in entsprechender Frequenz nachgewiesen werden können. Anders als unifikationsbasierte Ansätze gehen gebrauchsbasierte Ansätze davon aus, dass aus dem Sprachgebrauch heraus Konstruktionen als kognitive Repräsentationen entstehen und in einem Netzwerk geordnet sind. Gebrauchsbasierte Ansätze sind bemüht, Konventionalisierungsprozesse und damit die Etablierung und Stabilisierung von Konstruktionen durch ihren Gebrauch zu erfassen und möglichst adäquat zu beschreiben. Diesen Annahmen ist die Analyse zum Verb wirken als Ausschnitt aus einer breiter angelegten Studie zu nonagentiven Konstruktionen verpflichtet. Dabei implementiert sie, knapp gesagt, die auf die von Polenz'sche Sat:semantik (in der aktuellen Auflage von 2008) zurückgehenden Konzepte Aussagerahmen, Prädikationsrahmen, Prädikatsklassen und semantische Rollen in die Goldberg'sche Struktur einer Konstruktion, ${ }^{13}$ um ein tragfähiges Modell zur Analyse von Konstruktionen (und vor allem von Konstruktionsbedeutungen) vorzuschlagen und diese in einem Konstruktikon, das semantische und formale Beziehungen berücksichtigt, zu ordnen. Zugleich wird in der Analyse auf die Erklärungspotentiale

11 E-Valbu, <http://hypermedia2.ids-mannheim.de/evalbu/>, Stand: 04.03.2014.

12 Die Vorarbeiten in den 80er Jahren sind für den Ansatz Goldbergs und die aktuellen Studien relevant (vgl. Fillmore 1988, 1989; daneben Lakoff 1987; Langacker 1982, 1987, 1988 und 1991). Zum aktuellen Stand vgl. exemplarisch Goldberg (2006), Langacker (2005, 2009), Croft \& Cruse (2004) sowie Croft (2013).

13 Peter von Polenz schloss seine früh publizierte Satzsemantik (erstmals 1985) direkt an Fillmore (1968) an. Vgl. dazu ausführlich die Hinführung und Entwicklung des Modells, wie es für die Studie Nonagentive Konstruktionen des Deutschen, die an der CAU zu Kiel als Habilitationsschrift entsteht, entwickelt wurde und bereits in Ziem \& Lasch (2013: 110-142) auszugsweise vorgestellt wird. Zur Relevanz des Konzeptes für die Beschreibung sprachlicher Strukturen vgl. weiter zuletzt Busse (2012: 522ff.). 
syntaktischer Funktionen verzichtet, die Goldberg $(1995,2006)$ und Croft (2001) noch in die Beschreibung der Struktur von Konstruktionen (auch in Tradition Fillmores 1988) einbeziehen.

\section{Das Verb wirken in der Konstruktion der Askription}

Nonagentive Konstruktionen zeichnen sich durch eine spezifische Perspektivierungsleistung aus. ${ }^{14}$ Die Nominalphrase im Nominativ, die traditionell mit der Funktion Subjekt in satzwertigen Ausdrücken identifiziert wird, trägt hier nicht die semantische Rolle des Agens (AG), das wäre die zentrale Perspektive bei der Sprachproduktion, sondern ist beispielsweise als Patiens (PAT), Objekt (AOB bzw. EOB bzw. ein SOB) oder Benefaktiv (BEN) einzustufen. ${ }^{15}$ Damit nimmt ein Sprecher die zentripetale Perspektive bei der Sprachproduktion ein. Ausgehend von dieser Grundannahme sind nicht allein die Konstruktionen mit den so genannten Passivauxiliaren sein, bleiben, werden, bekommen/erbalten/kriegen zu beschreiben, sondern auch Konstruktionen mit scheinen, erscheinen, gebören, pflegen, drohen, stehen, gehen, wirken (aussehen, anbören usw.) und haben. In einem weiteren Schritt wären z. B. alle Instanzen der Konstruktionen [[NP] + [VP] + [ADJD]] in die Überlegungen einzubeziehen, die das Merkmal erfüllen, in der NP im Nominativ, funktional dem Subjekt, kein AG, sondern ein SOB einzubetten. Schließlich müsste noch geprüft werden, wie spezifiziert der Slot des Qualitativs (QUAL), ${ }^{16}$ in den hier Adjektive unterschiedlicher Form eingebettet sein können, ist (in die Konstruktion mit sein z. B. kann auch ein am-Progressiv eingebettet werden usw.). Explizit sei betont, dass im Folgenden nicht davon ausgegangen wird, dass das Adjektiv mit einem ,Passivauxiliar' einen ,verbalen Komplex‘ bilde (vgl. z. B. wenn auch bereits abgeschwächt von Polenz 2008: 184-186). ${ }^{17}$

Drei zentrale Konstruktionstypen der nonagentiven Konstruktionen sind zu unterscheiden und bilden einen engeren Zusammenhang im Konstruktikon: die Konstruktionen der (1) Askription, (2) der Kommutation und (3) der Akzeptation; letztgenannte Konstruktionen werden in diesem Beitrag ausgeklammert. ${ }^{18}$ Mit dem Begriff der Askription (aus

14 Grundlegend Köller (1997); seine Ausführung zum Passiv (als Konverse) (ebd. u. a. 468) teile ich nicht.

15 Zu den semantischen Rollen vgl. von Polenz (2008: 167-174), mit Einschränkungen Primus (2012), weiter Busse (2012) sowie Ziem \& Lasch (2013: 124-127), hier zur Präzisierung, Erweiterung und Diskussion vor allem 125: Ein affiziertes Objekt (AOB) ist eine „,von einer Handlung oder einem Vorgang betroffene Person oder Sache“, ein effiziertes Objekt (EOB) eine „durch eine Handlung oder einen Vorgang entstehende Person oder Sache“ und das spezifizierte Objekt (SOB) eine „,durch eine Eigenschaftszuweisung (z. B. mittels eines QUAL) spezifizierte Person oder Sache“.

16 Der Qualitativ (QUAL) gibt eine Eigenschaft von etwas an. Zur Diskussion des Postulats einer eigenen semantischen Rolle, die den Verbalkomplex (Kopula und Prädikativ) faktisch zu Gunsten einer abstrakteren Konstruktion auflöst, vgl. Ziem \& Lasch (2013: 125 mit Anm. 82).

17 Wie von Polenz (1985; im Nachdruck 2008) gehen auch Ackermann \& Webelhuth (1998) (leider) in einer recht frühen konstruktionsgrammatischen Analyse zum Passiv im Deutschen (219-267) von der „Konverse“ aus, die heute im Hinblick auf die Ausweitung auf, passivähnliche“ Phänomene nicht mehr zu halten ist.

18 Der Begriff der Kommutation aus lat. commutatio (,Wandel, Wechsel') steht für die Markierung, dass ein affiziertes Objekt (AOB) eine Umwandlung oder Veränderung erfährt/erfahren soll: Das Fenster wird/gehört geschlossen. Konstruktionen der „Akzeptation“ aus lat. ac-cipio (,hinnehmen, entgegennehmen, abnehmen, in Empfang nehmen, sich geben-, sich reichen lassen'; ,empfangen, annehmen, billigen') setzen einen Benefaktiv (BEN) in den Fokus: Er bekommt den Führerschein entzogen (vgl. dazu aus kognitionslinguistischer, nicht konstruktionsgrammatischer Sicht mit ganz ähnlichem Anliegen vor allem Lenz 2013). Eine besondere Stellung nehmen schließlich die Konstruktionen mit baben ein, die bspw. Eroms als „Haben-Passiv“ zum „Dativpassiv“ stellt (Eroms 2000: 420f.). Aus konstruktionsgrammatischer Perspektive bilden diese Konstruktionen (Der Baum hat in seine Rinde ein Herzeingeritzt) eine Brücke zwischen den Konstruk- 
dem lat. $a$-scrïbo ,bei-, zuschreiben, schreibend beifügen, hinzusetzen` abgeleitet) wird in der Sprachwissenschaft ein Wortkomplex bezeichnet, der ein Objekt - nicht formal syntaktisch verstanden - identifiziert und ihm eine Bedeutung zuordnet. In diese sind u. a. mit je spezifizierten Qualitativen eingebettet die Verben sein, bleiben, scheinen, erscheinen, pflegen, drohen, stehen, gehen, wirken usw.

(5) Das Fenster ist geschlossen.

Verben der ASKRIPTION (Subtyp der Prädikationsklasse ZUSTAND) mit sein:

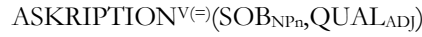

Verben des Aussagerahmens ,Eigenschaftszuweisung' können in den Prädikationsrahmen ASKRIPTION (als Subtyp der Prädikatsklasse der ZUSTANDs-Verben) ${ }^{19}$ eingebettet sein und dabei hinsichtlich ihrer Bedeutung in einem spezifischen Verhältnis zur Konstruktionsbedeutung stehen. Folgende Relationen werden in Präzisierung von Goldberg (1995: 59-66) postuliert: ${ }^{20}$

Die Verbbedeutung interagiert direkt mit der Konstruktionsbedeutung:

- Direkte Relation (= direkt): Verb- und Konstruktionsbedeutung, die durch Prädikations- und Aussagerahmen ausgedrückt sind, entsprechen einander.

Die Verbbedeutung interagiert indirekt mit der Konstruktionsbedeutung:

- $\quad$ Modale Relation $(\approx$ modal): Die Art und Weise einer (konzeptualisierten) Handlung oder eines (konzeptualisierten) Vorgangs wird in den Vordergrund gerückt. Auch eine Sprecher_inneneinstellung kann markiert sein.

- $\quad$ Resultative Relation ( $\approx$ resultativ): Das Resultat einer (konzeptualisierten) Handlung oder eines (konzeptualisierten) Vorgangs wird in den Mittelpunkt gestellt. ${ }^{21}$

- Intendiertes Resultat $(\approx$ intendiertes Resultat): Das intendierte Resultat einer (konzeptualisierten) Handlung oder eines (konzeptualisierten) Vorgangs wird in den Mittelpunkt gestellt.

Sein interagiert direkt mit der angenommenen Konstruktionsbedeutung (=). An Argumenten sind in der Konstruktion vorgesehen ein spezifiziertes Objekt (SOB) und ein Qualitativ (QUAL). In diese Slots, also die Argumentstellen der Konstruktion, können unterschiedliche Filler eingebettet werden: Während das SOB auf formaler Seite meist durch eine Nominalphrase im Nominativ repräsentiert ist, ist der QUAL hinsichtlich seiner Spezifizierung freier, im Beispiel wird ein deverbales Adjektiv (auf der Basis eines Partizip II) eingebettet. Alle Argumentstrukturrollen der Konstruktion und Partizipantenrollen des Verbs sein fusionieren, da in den Rollenplänen sowohl auf Seiten der Konstruktion wie auf Seiten des Verbs die semantischen Rollen des SOB und des QUAL vorgesehen sind. ${ }^{22}$

tionen der Askription und der Akzeptation. Diese kann allerdings nur angenommen werden, wenn kontextsensitiv semantische Rollen in den jeweiligen Realisierungen von Konstruktionen bestimmt werden.

19 Vgl. zu den Prädikationsklassen und -rahmen von Polenz (2008: 159). Zur Adaption vgl. Ziem \& Lasch (2013: 122124).

20 Die Relationen sind nicht als geschlossene Reihe zu verstehen. Vgl. dazu ausführlich meine Überlegungen in Ziem \& Lasch (2013: 129f.).

21 Es sei betont, dass eine strenge Zuordnung der semantischen Rollen nicht möglich ist bzw. von Fall zu Fall vorgenommen werden muss. Neben dem SOB könnte für die resultative Relation sowie die des intendierten Resultats auch ein EOB postuliert werden.

22 Zur Fusion vgl. Goldberg (1995: 50-52), Evans \& Green (2006: 671-680) sowie den Überblick in Smirnova \& Mortelmans (2010). 


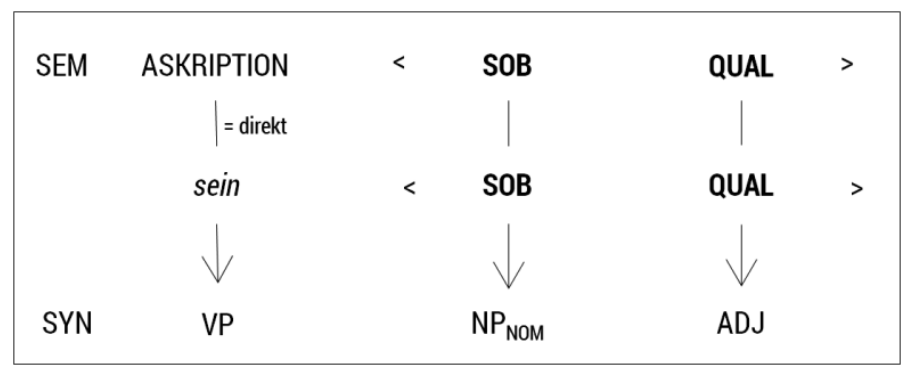

Abbildung 1: Konstruktion der ASKRIPTION (Subtyp der Prädikationsklasse ZUSTAND) mit $\operatorname{sein}^{23}$

Implementiert man diese Analyse des Prädikationsrahmens ASKRIPTION in das von Goldberg vorgestellte Schema der Struktur einer Konstruktion ${ }^{24}$ und passt dieses an die hier gemachten Annahmen an, dann lässt sich die Struktur der Konstruktion wie in Abbildung 1 darstellen. Fragen wir nach dem Verb wirken in nonagentiven Konstruktionen der Askription, dann ist hier vorerst nur das Strukturformat relevant, das gerade am Beispiel mit sein illustriert wurde. ${ }^{25} \mathrm{Wie}$ wir sehen werden, wird wirken in zwei Gebrauchsvarianten in die Konstruktion der Askription eingebettet, die je gemeinsam mit spezifischen Qualitativen auftreten. Wirken mit deverbalem Adjektiv auf der Basis eines Partizip I lizenziert verschiedene Optionen, die zunächst abhängig vom Grad der Agentivität des in der Nominalphrase im Nominativ Codierten scheinen. Lewin und das Medikament stellen dabei exemplarisch die Pole eines mehrdimensionalen Agensbegriffs dar. ${ }^{26}$

(6) a. ?Lewin wirkt hemmend.

b. Das Medikament wirket hemmend.

Der Status von wirken in (6) ist keinesfalls eindeutig. Wenn man (6a) nicht gänzlich als ungrammatisch einstuft, hat man keine andere Wahl, als (6a) in agentiver Lesart aufzufassen (,die momentane Aktivität von Lewin hemmt'), weshalb (6a) hier als agentive Konstruktion mit Lewin als Agens ausgeklammert werden kann. Es verweist auf einen anderen Ausschnitt des Konstruktikons, zu dem sich Beziehungen herstellen lassen über eine Konstruktion niedrigeren Abstraktionsgrades, sprich: bspw. das Verb wirken und seine unterschiedlichen Gebrauchsvarianten. (6b) hingegen ist im Sinne der Konstruktion der Askription akzeptabel: ,Das Medikament hat die Eigenschaft, hemmend zu wirken`. Man ist geneigt, den Agentivitätsgrad von Medikament als Grund für die Begünstigung der nona-

23 Zur Bezeichnung der Rollen, die durch das Verb beigesteuert werden, könnte hier auch, um die valenzgrammatische Relevanz herauszuheben und Konstruktions- und Verbbedeutung auf den ersten Blick schärfer zu trennen, die Umschreibung der Rollen genutzt werden, die beim elektronischen Valenzwörterbuch E-VALBU Verwendung finden (<http://hypermedia2.ids-mannheim.de/evalbu/>, Stand: 04.03.2014).

24 Dieses sei hier ausgewählt, da sich die meisten gebrauchsbasierten Ansätze mehr oder weniger explizit immer wieder auf Goldberg berufen und daher die diagrammatische Darstellung der Konstruktionsbedeutung auch für diese Studien sich als kommunikativ anschlussfähig erweisen dürfte.

25 Sein tritt, wie bereits eingangs erläutert (Abschnitt 2), mit anderen Konstruktionen auf. Zu Gunsten einer stringenten Darstellung mit Fokus auf das Verb wirken werden diese in diesem Artikel ausgeklammert.

26 Vgl. den mehrdimensionalen Agensbegriff nach Lakoff (1977) und Dowty (1991). Danach sind die Dimensionen der Agentivität: Volitionalität (Intentionalität), Verursachung, Sentience (Erfahrung) sowie Bewegung. Das Medikament kann danach zwar als Verursacher einer Wirkung konzeptualisiert werden, aber viel stärker noch als Spezifiziertes Objekt, da es weder belebt ist, noch die Wirkung intentional zu verursachen in der Lage ist. 
gentiven Lesart zu sehen. Voraussetzung dafür wäre, dass zumindest eine Rolle, die die Konstruktion der Askription zur Verfügung stellt, mit den Rollen, die das Verb einbringt, fusioniert (hier das SOB). Allerdings - dies ist eine gewichtige Einschränkung - sieht man in diesem Beispiel noch die Vollverbsemantik von wirken im Sinne von (6a) durchscheinen. Letzteres lässt sich strukturell belegen: Liest man (6b) in der vorgeschlagenen Art, interpretierte man wirken in (6b.) als kopulaähnliches Verb. Für diese soll aber in der Regel gelten, dass das „Partizip I [...] nicht als Prädikativ bei einem Kopulaverb (sein, werden, bleiben) oder einem Kausativverb (machen, lassen) stehen [kann], außer es hat sich zu einem eigenständigen Adjektiv entwickelt“ (Duden-Grammatik 2009: 358) - streng genommen müsste man also deshalb wirken in (6) in beiden Fällen als Instanzen agentiver Konstruktion einstufen. Das wird auch durch den konstruktionalen Status des Partizips I hemmend unterstützt. Für das Partizip I hat Welke (2005: 196) in einem anderen Kontext plausibel zeigen können, dass es Merkmale der Imperfektivität trägt und deshalb aktivische perspektivierende Eigenschaften trägt, die aus den aspektuellen folgen. Das hat zur Konsequenz, dass, selbst wenn man hemmend als Adjektiv auffasst, dieses deverbale Adjektiv (auf der Basis des Partizip I) u. a. „die Art und Weise eines Ereignisses oder eines Zustandes beschreib[t]“ (Duden-Grammatik 2009: 358) und damit funktional als Adverbial in satzwertigen Ausdrücken interpretiert werden kann. Setzt man die Beispiele ins Perfekt wie in (7), wird das Dilemma hinsichtlich der Einstufung des Status von wirken noch deutlicher:

(7) a. ?Lewin hat hemmend gewirkt.

b. Das Medikament hat hemmend gewirkt.

Man kann beide Beispiele in (7), wiederum mit den Einschränkungen für (7a), in agentiver Lesart auffassen, wofür, wie gesagt, die Annahme eines spezifischen Agentivitätsgrades von Lewin und Medikament jedoch Voraussetzung zu sein scheint. Die nonagentive Lesart in (7b) wird, anders als im Präsens, hier noch stärker blockiert, da wirken das Perfekt mit baben und nicht mit sein bildet. ${ }^{27}$ Die Optionen, die (6b) und (7b) im Hinblick auf die Einbettung von wirken mit Partizip I in nonagentive Konstruktionen dennoch offerieren, sind möglicherweise ein Reflex auf die Etablierung von wirken und deverbalem Adjektiv auf der Basis des Partizips II in Konstruktionen der Askription. Nimmt man an, dass in (6b) und (7b) diese nonagentive Lesart als Möglichkeit noch ausstehender Grammatikalisierungsprozesse ernst zu nehmen (und zumindest nicht gänzlich auszuschließen) ist, könnte man die Struktur der Konstruktion für diese Fälle (schon jetzt) wie folgt darstellen. In der Korpusanalyse (Abschnitt 4) werden u. a. die Bedingungen für diese Variante erarbeitet.

27 Vgl. auch dazu grundsätzlich Welke (2005). 


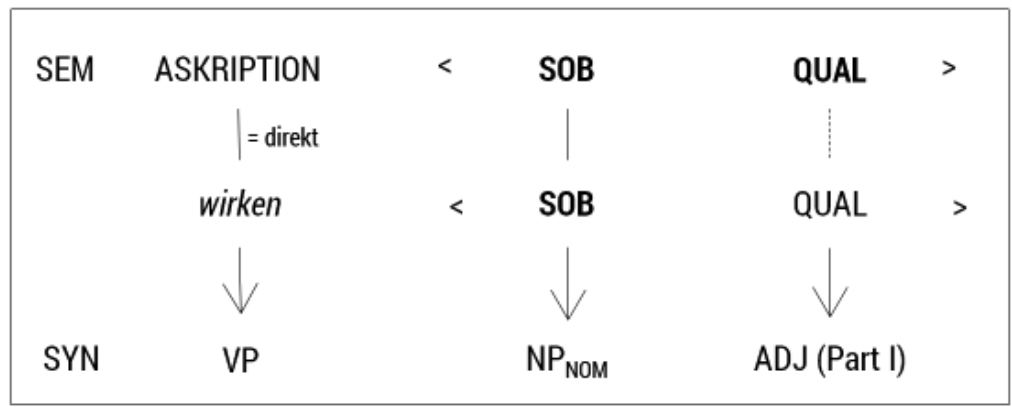

Abbildung 2: Konstruktion der ASKRIPTION (Subtyp der Prädikationsklasse ZUSTAND) mit wirken und deverbalem Adjektiv (Partizip I) mit direkter Relation zwischen Verb- und Konstruktionsbedeutung

Anders als bei sein ist bei wirken in dieser Gebrauchsvariante kein QUAL im Rollenplan angelegt. Verb- und Konstruktionsbedeutung können nur über die Argumentstrukturrolle und Partizipantenrolle des SOB fusionieren; der QUAL wird durch die Konstruktion lizenziert. ${ }^{28}$ Das ist zum einen der Grund für die strittige Beurteilung von wirken als Kopula (und damit zugleich der Grund, warum das deverbale Adjektiv in der Form des Partizips I hier stehen kann), zum anderen für die ambige Beurteilung der Lesart eines Satzes wie (6b) und zum dritten Indiz dafür, dass ein Grammatikalisierungsprozess noch nicht abgeschlossen ist, der semantische Potentiale des Verbs wirken (analog zum so genannten Halbmodal scheinen) weiter abbaut, ${ }^{29}$ wie bei der Einbettung von Adjektiven auf der Basis des Partizips II zu sehen sein wird.

Eine gänzlich andere Interpretation stellt sich dann nämlich ein, wenn ein deverbales Adjektiv auf der Basis eines Partizips II als QUAL in die Konstruktion eingebettet wird, wie in 8 .

(8) a. Lewin wirket gehemmt.

b. *Das Medikament wirket gehemmt.

Wirken und Partizip II sind in (8a), unabhängig vom Agentivitätsgrad des Elements, das in der Nominalphrase im Nominativ codiert ist, nicht mehr in einem Handlungs- oder Vorgangszusammenhang verstehbar, sondern müssen als hinsichtlich der Faktizitätsbehauptung durch einen Sprecher markierte Eigenschaftszuweisung aufgefasst werden. Grund dafür ist auch die Konstruktionsbedeutung, die das Partizip II mit in die Konstruktion der Askription einbringt. Welke (2005) bezeichnet nach umfangreicher Diskussion unterschiedlicher Forschungspositionen das Partizip II als "Spiegel des Perfekts“ und meint damit, dass der „Zustand als Resultat eines vorangegangenen Vorgangs (Nachzustand) [...] die archetypische Bedeutung des Partizips II perfektiver Verben“ (238) sei. Im Vergleich zum Partizip I trage es das Merkmal der Perfektivität und sei damit für die passivische Perspektivierung prädestiniert (196). (8b) hingegen scheint nicht akzeptabel zu sein; Fälle wie dieser werden vor dem Hintergrund der kollokativen Bindung zwischen der als SOB eingebetteten Nominalphrase und dem QUAL in der Korpusanalyse (Abschnitt 4) noch einmal diskutiert. Wirken in (8a) verhält sich hier in der Konstruktion der Askription voll-

28 Das wird im Schaubild durch die gestrichelte Linie und die nicht durch Fettdruck hervorgehobene Rolle des QUAL auf Ebene des Verbs visualisiert.

29 Vgl. dazu Eisenberg (2006 II: 364). 
kommen strukturanalog zu scheinen und erscheinen, um nur zwei der Möglichkeiten zu nennen, in denen die Relation zwischen Bedeutung der Konstruktion der Askription und Verbbedeutung als modal zu beschreiben ist. Die Bedeutung der Konstruktion der Askription, der Eigenschaftszuweisung, wird in dieser Relation dahingehend aktualisiert, dass die Faktizitätsbehauptung des Sprechers, der die Konstruktion realisiert, modalisiert wird.

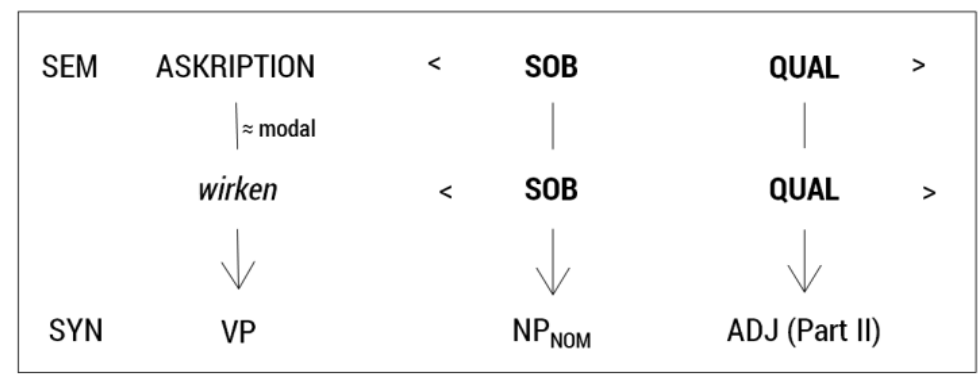

Abbildung 3: Konstruktion der ASKRIPTION (Subtyp der Prädikationsklasse ZUSTAND) mit wirken und deverbalem Adjektiv (Part II) mit modaler Relation zwischen Verb- und Konstruktionsbedeutung

Ich habe mich hier aufgrund der Strukturanalogie von scheinen und erscheinen in der Konstruktion der Askription (und damit gegen Eisenberg 2006 II) dazu entschieden, bereits dafür zu plädieren, dass die Rollen des SOB und des QUAL fusionieren und damit wirken auf einer Stufe mit scheinen und erscheinen steht. Anders als wirken variieren diese allerdings hinsichtlich einer anderen Kategorie, nämlich des Tempus. ${ }^{30}$ Je nach verwendetem Partizip als deverbalem Adjektiv und mutmaßlich in Abhängigkeit zum Agentivitätsgrad des in der Nominalphrase im Nominativ Codierten liegen Realisierungen unterschiedlicher Konstruktionen vor: Von Konstruktionen der Askription kann in dieser ersten Annäherung mit Sicherheit nur dann gesprochen werden, wenn neben wirken ein deverbales Adjektiv auf der Basis eines Partizips II als Qualitativ in die Konstruktion eingebettet ist; strittig hingegen bleibt die Interpretation dann, wenn ein Adjektiv auf der Basis eines Partizips I in die Konstruktion eingebettet wird. Ein ähnlich ambiges Bild zeigt sich, wenn ein Adjektiv (als QUAL) zusammen mit wirken auftritt, wie in (9):

(9) a. Lewin wirket rastlos.

b. Das Medikament wirket schnell.

$\mathrm{Zu}$ diskutieren wäre an diesem Beispiel, ob man hier ohne Kontextinterpretation überhaupt noch zweifelsfrei eine Lesart, z. B. eine Realisierung einer Konstruktion der Askription, favorisieren kann - die Adjektive rastlos und schnell können in beiden Fällen nämlich zum einen funktional als Adverbial beschrieben werden, ${ }^{31}$ wenn man Lewin und das Medi-

30 Scheinen wird in der Konstruktion der Askription für Aussagezusammenhänge in Präsens und Imperfekt anscheinend präferiert (Das Fenster scheint geschlossen), erscheinen für analytische Vergangenheitstempora. Eine Erklärung könnte darin liegen, dass scheinen diese mit baben bildet. Einer der sehr wenigen (ungrammatischen aber sympathischen) Belege aus dem DWDS bei kleinschrittiger Abfrage (vgl. dazu analog Abschnitt 4) ist: Dieser Gedanke bat so plausibel geschienen [...] aus Mauthners Wörterbuch der Philosophie von 1910. Im Gegensatz dazu selegiert das perfektive erscheinen das Verb sein (exemplarisch aus Hans Delbrücks Kriegskunst im Rabmen der politischen Geschichte von 1900: [...] daß mir Helbigs Beweisführung nicht zwingend erschienen ist [...]) und übernimmt die Funktion von scheinen für die analytischen Vergangenheitstempora. Detailuntersuchungen sind u. a. Thema der Studie zu den nonagentiven Konstruktionen des Deutschen.

31 Abgesehen davon, dass der Status von wirken nicht geklärt ist, kann die Wortartendiskussion in diesem Bereich ebenfalls als - neutral gesagt - heterogen betrachtet werden. Eisenberg plädiert dafür, in solchen Fällen von einem ,adver- 
kament als agentiv einstuft oder wirken zumindest als Verb des VORGANGS - wenn nicht gar als HANDLUNG - konzeptualisiert. Zum anderen ist es möglich, für (9a) analog zum Beispiel (8a) und für (9b) analog zum Beispiel (7b) eine nonagentive Lesart zu postulieren, in der sich in der Konstruktion der Askription der QUAL auf das SOB bezieht (vgl. Abbildung 3) und nicht als Adverbial eine andere (syntaktische) Funktion im satzwertigen Ausdruck übernimmt (analog zu 6a und b) und damit Indikator für die Instanz einer anderen Konstruktion ist, die einen Knoten im Bereich der agentiven Konstruktionen des Konstruktikons bildet.

Wie an den diskutierten Beispielen bis hierhin deutlich geworden sein sollte, profitieren Konstruktionsgrammatik und Valenzgrammatik direkt voneinander: Ohne verschiedene Angaben des Gebrauchs von wirken und damit ohne die Rollenvorgaben, die das Verb (je nach Bedeutung) macht, ist eine systematische Beschreibung des Zusammenhangs von Konstruktions- und Verbbedeutung nicht möglich. Zum anderen wird gerade mit dem Format der Konstruktion der strukturell übergreifende Zusammenhang zwischen sprachlichen Einheiten in einem Konstruktikon deutlich - hier kann auch die Framesemantik weitere Einsichten bieten, die an anderer Stelle in diesem Band behandelt werden (vgl. die Artikel von Boas und Ziem).

Im Mittelpunkt der Analyse werden nach dieser ersten Annäherung auf der Basis ausgewählter Beispiele die zweifelsfreien Fälle der Konstruktion der ASKRIPTION (Subtyp der Prädikationsklasse ZUSTAND) mit wirken und deverbalem Adjektiv (auf der Basis des Partizips II) mit modaler Relation zwischen Verb- und Konstruktionsbedeutung (vgl. Abb. 3) wie in (10) stehen:

(10) Das Fenster wirkt geschlossen.

Es werden hier folgende Thesen vertreten:

1. Nicht die Partizipien perfektiver (telischer) oder imperfektiver (atelischer) Verben treten mit einem Kopula- oder Hilfsverb in Beziehung, sondern ein quantifizierbares Set an (deverbalen) Adjektiven (hier: geschlossen) kann in Konstruktionen der Askription zusammen mit einer Gruppe von Verben eingebettet werden. Die Wahl wird dabei nicht durch das verwendete Verb (hier: wirken), sondern durch die Konstruktion gesteuert.

2. Die Verben (hier: wirken), die in die Konstruktion der Askription eingebettet werden, interagieren mit der Konstruktionsbedeutung auf unterschiedliche Weise (hier mit deverbalem Adjektiv auf der Basis des Partizips II: modal). Sie tragen damit einen eigenen Bedeutungsanteil zur aktualisierten Konstruktionsbedeutung bei, der nicht vorhersagbar ist.

3. Die dergestalt aktualisierten Konstruktionen bilden einen Subtyp entlang der Relationen zwischen Konstruktionsbedeutung und Verbbedeutung, die von der Konstruktionsbedeutung her zu strukturieren sind:

Das Fenster ist geschlossen. sein als Verb der ASKRIPTION (Subtyp der Prädikationsklasse ZUSTAND): ASKRIPTIONV(= direkt)(SOBNPn, QUALADJ)

Das Fenster wirkt geschlossen.

scheinen, erscheinen und wirken [...] als Verben der ASKRIPTION (Subtyp der Prädikations-

bialen Adjektiv“ (Eisenberg 2006 II: 223) zu sprechen, andere stufen das Adjektiv sogleich funktional als Adverb ein. Eisenberg (2006 II; zum gesamten Zusammenhang und der Forschungsdiskussion vgl. 223-230) ist hier allein schon deshalb zu folgen, weil formale und funktionale Aspekte in der Analyse nicht vermengt werden sollen oder man keine Diskussion um den Status der Wortart Adverb führen kann, wie hier. 
klasse ZUSTAND): ASKRIPTIONV( $\approx$ modal) $\left(\mathrm{SOB}_{\mathrm{NPn}}, \mathrm{QUAL}_{\mathrm{ADJ}}\right)$

Sie sind konventionalisiert und damit in maschinellen Analysen seriell nachweisbar.

4. Die Konstruktionen der Askription und ihre Subtypen stellen einen Ausschnitt des Konstruktikons dar und stehen über in sie eingebettete Konstruktionen niedrigerer Hierarchiestufe (Nominalphrasen im Nominativ: Fenster, Verb: wirken, [deverbales] Adjektiv: geschlossen) mit anderen Bereichen des Konstruktikons in Beziehung (z. B. zu Konstruktionen der Transformation [das Fenster wird geschlossen] oder agentiven Konstruktionen [Lewin hat das Fenster geschlossen]).

\section{Nonagentive Konstruktionen mit wirken - eine qualitative Analyse}

Korpusgestützte Arbeiten zur deutschen Gegenwartssprache können sich leider nur auf wenige Korpora stützen, die außerdem nur in Ausnahmen Analysen zum Sprachgebrauch des 20. Jahrhunderts ermöglichen. Eines dieser Korpora ist das annotierte Kernkorpus beim Digitalen Wörterbuch der Deutschen Sprache (DWDS). ${ }^{32}$ Es umfasst insgesamt über 100 Millionen Wortformen, deckt die Sprachentwicklung des 20. Jahrhunderts (weitestgehend lückenlos) ab und ist geschichtet nach verschiedenen Kommunikationsbereichen: Neben belletristischen Texten (28,42\%) wurden Zeitungstexte (27,36\%), wissenschaftliche Veröffentlichungen (23,15\%) und „Gebrauchsliteratur“ (21,05\%) berücksichtigt. ${ }^{33}$ Der Umfang des Korpus macht es zwingend erforderlich, mit systematischen Abfragen zu arbeiten, da eine Zufallsauswahl aus Belegen, die mit einfachen Suchroutinen ermittelt wurden, die Ergebnisse unnötig verzerrt. ${ }^{34}$ Für die systematische Erhebung von Daten für eine Analyse der Konstruktionen der Askription aus dem Kernkorpus wurden deshalb eigene Routinen entwickelt, die eine Klassifikation der Belege hinsichtlich verschiedener Variablen halbautomatisch erlauben. Von diesen Parametern ist in diesem Beitrag die Berücksichtigung der Variationen der (teils unsystematischen) Annotation im Hinblick auf die Besetzung des Qualitativs (geschlossen kann als VVPP [Partizip] oder als ADJD [Adjektiv] im Korpus annotiert sein) relevant. ${ }^{35}$

Die Komplexität des Datenmaterials setzt diese feine Differenzierung voraus, um belastbare Aussagen über die Entwicklung von Konstruktionen in größeren Zeitläufen machen zu können. Im Folgenden werde ich mich auf die Erhebungsroutinen zur Konstruktion der ASKRIPTION (Subtyp der Prädikationsklasse ZUSTAND) mit wirken + (deverbalem) Adjektiv anhand des Präsens Indikativ und der Besetzung des QUAL durch

32 Das DWDS steht online zur Verfügung unter < http://www.dwds.de/>, Stand: 04.03.2014.

33 Die Prozentangabe der Schichtung ist der detaillierten Beschreibung des Kernkorpus entnommen. Diese steht unter <http://www.dwds.de/ressourcen/kernkorpus/>, Stand: 04.03.2014, zur Verfügung.

34 Auch wenn keine exhaustive Analyse angestrebt werden kann, so ist doch die Abbildung einer Entwicklungstendenz bestimmter Realisierungen von Konstruktionen wünschenswert. Vgl. dazu die Kritik von Lasch (i. Dr.) an Felfe (2012).

35 Da in diesem Beitrag nur das Verb wirken im Mittelpunkt steht, hat diese halbautomatische Erfassung hier weniger Relevanz als in noch ausstehenden Vergleichen zu anderen Verben wie scheinen oder erscheinen. Differenziert wird dort dann weiter nach Kommunikationsbereich (BE=Belletristik, ZE=Zeitungstext, WI=Wissenschaftliche Publikation, $\mathrm{GE}=$ Gebrauchsliteratur) und Veröffentlichungszeitpunkt (Feindifferenzierung nach Erscheinungsjahr und -dekade), Tempus der Verbalkonstruktion mit Berücksichtigung der Doppelperfektformen (wirkt geschlossen; bat geschlossen gewirket gehabt usw.) als inhärente Kategorie (Modus wird nicht berücksichtigt), Person und Numerus der Verbalkonstruktion (wirkt/wirken gescblossen usw.) als Kohärenzkategorien, Stellung des finiten Teils der Verbalkonstruktion zur gezielten Abfrage der Position im Stellungsfeld satzwertiger Ausdrücke (das Fenster wirkt geschlossen vs. [...], dass das Fenster geschlossen wirkt). 
Partizip II (in der Annotation: VVPP) und Adjektiv (ADJD) konzentrieren (vgl. Abb. 4). ${ }^{36}$ Wie in der Übersicht zu sehen ist, wird jede hier relevant gesetzte Variable durch einen eigenen Suchstring geprüft, wobei Überschneidungen durch Formidentität berücksichtigt sind. Zur Veranschaulichung werden die Zeilen 10 und 12 der Übersicht eingehender beschrieben. Die Verbalkonstruktionen in $1 \mathrm{P}$ und $3 \mathrm{P}$, Plural, Präsens sind formidentisch und damit auch die Abfrageroutine: „\$p=VVPP \#0@wirken“ \&\& !werden with \$p=VAINF \&\&!@zu“. Gesucht wird nach einem Partizip (VVPP) im Wortabstand 0 (\#0) zur morphologisch definiten Form wirken (@wirken) und (\&\&) unter Ausschluss (!) einer beliebigen morphologischen Variante von werden als Hilfsverb (VAINF) und einem erweiterten Infinitiv mit zu (@zu). Durch Ausschluss von werden als ,Hilfsverb“(VAINF) soll vermieden werden, Belege im Futur I mit abzufragen: Daten der Form [...], dass die Fenster geschlossen wirken werden erfasst; Daten im Futur wie [...], dass die Fenster geschlossen wirken werden ignoriert die Abfrage. Auch der Ausschluss des erweiterten Infinitivs mit $z u$ ist hier eine reine Vorsichtsmaßnahme in Analogie zum Ausschluss von werden, um die Ergebnisse nicht zu kontaminieren. ${ }^{37}$

36 Beziehen wir uns im Folgenden auf die Annotationskategorien, werden diese als VVPP und ADJD bezeichnet. Gleiches gilt für VAINF (Hilfsverb) und VVPS (Partizip I).

37 Die erweiterten Infinitive werden - auch wenn ein gemeinsames Auftreten mit wirken höchst unwahrscheinlich ist mit einer eigenen Routine abgefragt (,@zu \#0 \$p=VVINF \#0@wirken“ \&\& !werden with \$p=VAINF). 

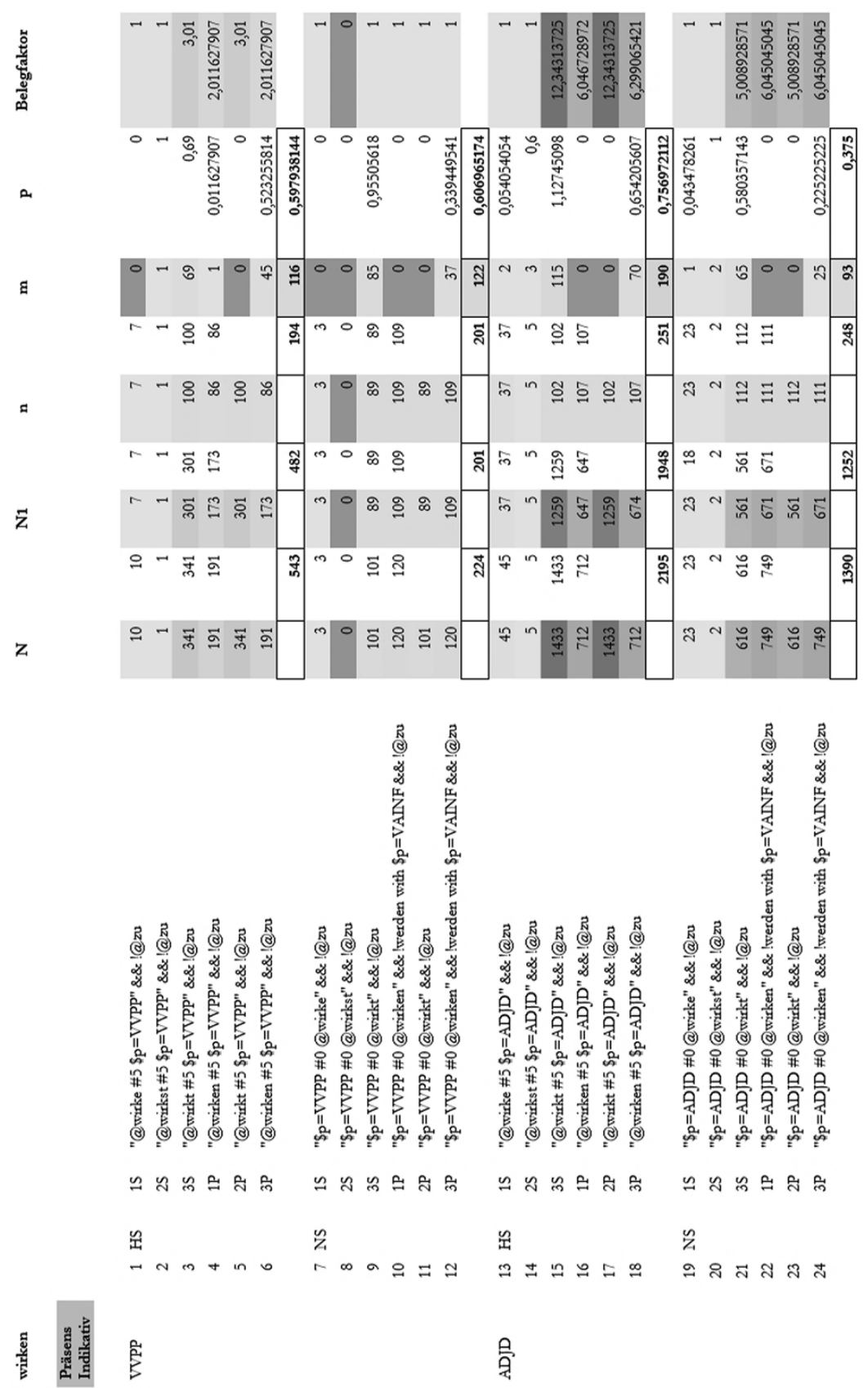

Abbildung 4: Abfrageroutinen und Belegzahlen für die Konstruktion der Askription mit wirken im Kernkorpus des DWDS (Präsens Indikativ, VVPP und ADJD) 
Die folgenden Spalten schließen die Belege für die Suchanfragen auf. N und N1 stehen für die ungesichteten Ergebnisse. Nicht alle Belege sind aufgrund rechtlicher Beschränkungen einsehbar $(\mathrm{N}=120)$. Deshalb bilden die zugänglichen Daten $(\mathrm{N} 1=109)$ die Basis der Auswertung ( $\mathrm{n}=109)$. Nach der Sichtung und Auswertung der Belege ergibt sich, dass keine Konstruktionen für 1P im Plural vorliegen ( $\mathrm{m}=0$ in Zeile 10), dafür aber 37 Belege für die 3P im Plural ( $\mathrm{m}=37$ in Zeile 12). Das Verhältnis zwischen erhobenen und für die Fragestellung relevanten Belegen gibt $\mathrm{p}$ an (gerundet $\mathrm{p}=0,339)$ - dieses Verhältnis wird $\mathrm{u}$. a. dafür genutzt, um die Ergebnisse zu interpolieren in Bezug auf die Gesamtgröße des Korpus und alle mit dem String abgefragten Belege $(\mathrm{N}=120)$. Der Belegfaktor gibt schließlich das Verhältnis zwischen zugänglichen Daten (N1) und in der Untersuchung berücksichtigen Belegen (n) an, um zumindest tendenzielle Aussagen über die Belegverteilung im gesamten Korpus machen zu können: Für den beschriebenen String liegt der Belegfaktor $\mathrm{N} 1 / \mathrm{n}$ bei 1 , da alle abgefragten Daten analysiert worden sind. Anders stellt sich dies für die Abfrage mit ADJD in den Zeilen 22 und 24 dar: Hier werden insgesamt 749 Belege im Korpus aufgerufen $(\mathrm{N})$, von denen 671 (N1) zugänglich sind. Von diesen wird jeder sechste Beleg als Basis der Untersuchung $(\mathrm{n}=111)$ ausgewählt. 25 Belege kommen für unsere Fragestellung in die engere Wahl (m), das Verhältnis zwischen untersuchten und relevanten Belegen schließt $\mathrm{p}$ gerundet mit 0,225 auf. Der Belegfaktor (N1/n) ist hier ungleich höher und wird später (wie p) wichtig, um Rückschlüsse auf die Verteilung der Belege im Gesamtkorpus ziehen zu können, indem er ins Verhältnis gesetzt wird zu N1 und N.

Doch auch dieses System der detaillierten Abfrage weist noch Defizite auf, die allerdings zum jetzigen Zeitpunkt keiner befriedigenden Lösung zugeführt werden können. Zum einen ist da die relativ geringe Spannweite der Abfrage für die Konstruktion bei Verbzweitstellung des finiten Teils der Verbalkonstruktion zu nennen. Mit einem Wortabstand von 5 (\#5; vgl. Zeilen 1-6 und 13-18) wird ein Teil der Belege nicht erfasst. Die Reduktion ist aber notwendig, um die Datenmenge überhaupt in den Griff zu bekommen - denn am Schluss muss jeder Beleg nach halbautomatischer Sortierung und ersten Sichtung noch interpretiert werden. Weiter werden Belege ausgeschlossen, in denen $z u$ als Gradpartikel fungiert: Er wirkt zu verschlossen. Allerdings ist die Belegzahl hier verschwindend gering und im Vergleich zum systematischen Ausschluss des erweiterten Infinitivs in Kauf zu nehmen. Einzig der Beleg (11) würde in der Untersuchung berücksichtigt werden: ${ }^{38}$

(11) $1998|\mathrm{BE}|[\ldots]$, daß Sie zu ernst, zu trocken oder zu still wirken?

Alle anderen Belege, die mit diesem String abgerufen werden, würden verworfen. Das hat verschiedene Gründe. Ausgeklammert werden zum einen Realisierungen, in die Modalverben involviert sind. Zum einen ist durch Modalisierung eine weitere Spezifizierung und Komplexitätssteigerung der Konstruktionen der Askription zu erwarten. ${ }^{39}$ Zum anderen wird in den meisten Fällen durch die Verwendung des Modalverbs die Realisierung der Konstruktion mit Verbzweitstellung aufgerufen, die hier gerade nicht erfragt werden sollte. Zum dritten wird die gezielte Abfrage der Kategorie Tempus und Numerus durch Modalisierung unmöglich.

38 Fragt man gezielt Konstruktionen mit Gradpartikel zu ab, hätte man folgenden String zu verwenden: „@zu \#0 \$p=ADJD \#0@wirken“ \& \& !werden with \$p=VAINF.

39 Zum Modus und zu den Konstruktionen mit Modalverb (z. B. modalen Infinitiven) arbeiteten bisher aus konstruktionsgrammatischer Perspektive Knobloch (2005), Petrova (2008) und Stefanowitsch (2009). 


\section{VVPP (gesichtet)}

\begin{tabular}{|c|c|c|c|c|c|c|c|c|c|c|}
\hline & DO1 & $\mathrm{DO} 2$ & $\mathrm{DO}_{3}$ & D04 & D05 & DOG & D07 & D08 & DOS & D10 \\
\hline $\mathrm{BE}$ & 2 & 0 & 3 & 0 & 0 & 1 & 1 & 0 & 0 & 1 \\
\hline $\mathrm{GE}$ & 28 & 9 & 16 & 15 & 7 & 22 & 3 & 4 & 2 & 6 \\
\hline WI & 7 & 5 & 10 & 6 & 9 & 12 & 5 & 3 & 7 & 18 \\
\hline \multirow[t]{3}{*}{ ZE } & 11 & 7 & 7 & 3 & 0 & 0 & 0 & 1 & 2 & 5 \\
\hline & 48 & 21 & 36 & 24 & 16 & 35 & 9 & 8 & 11 & 30 \\
\hline & D01 & DO2 & D03 & D04 & D05 & DOG & DO7 & D08 & Do9 & D10 \\
\hline wirke & 0 & 0 & 0 & 0 & 0 & 0 & 0 & 0 & 0 & 0 \\
\hline wirkst & 0 & 0 & 0 & 0 & 0 & 1 & 0 & 0 & 0 & 0 \\
\hline wirkt & 30 & 15 & 26 & 15 & 10 & 25 & 6 & 6 & 8 & 13 \\
\hline \multirow[t]{2}{*}{ wirken } & 18 & 6 & 10 & 9 & 6 & 9 & 3 & 2 & 3 & 17 \\
\hline & 48 & 21 & 36 & 24 & 16 & 35 & 9 & 8 & 11 & 30 \\
\hline
\end{tabular}

$\begin{array}{lllllllllll}\text { Zuwachs } & 48 & 69 & 105 & 129 & 145 & 180 & 189 & 197 & 208 & 238\end{array}$

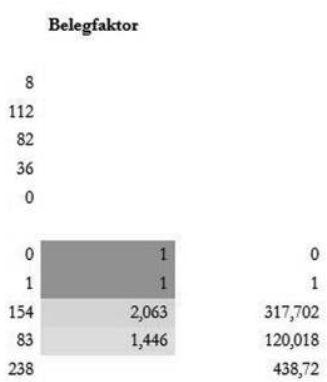

\begin{tabular}{|c|c|c|c|c|c|c|c|c|c|c|c|c|c|}
\hline & D01 & D02 & $\mathrm{D} 03$ & D04 & D05 & D06 & D07 & D08 & D09 & D10 & & & \\
\hline $\mathrm{BE}$ & 3 & 2 & 0 & 0 & 1 & 2 & 0 & 0 & 0 & 2 & 10 & & \\
\hline GE & 21 & 14 & 19 & 23 & 4 & 24 & 11 & 12 & 8 & 4 & 140 & & \\
\hline WI & 5 & 8 & 5 & 11 & 9 & 13 & 8 & 6 & 8 & 9 & 82 & & \\
\hline \multirow[t]{3}{*}{ ZE } & 11 & 6 & 9 & 8 & 2 & 3 & 3 & 1 & 1 & 7 & 51 & & \\
\hline & 40 & 30 & 33 & 42 & 16 & 42 & 22 & 19 & 17 & 22 & 283 & & \\
\hline & D01 & $\mathrm{D} 02$ & D03 & D04 & D05 & D06 & D07 & D08 & D09 & D10 & & & \\
\hline wirke & 1 & 0 & 0 & 1 & 1 & 0 & 0 & 0 & 0 & 0 & 3 & 1 & 3 \\
\hline wirkst & 1 & 1 & 0 & 1 & 0 & 0 & 0 & 1 & 0 & 1 & 5 & 1 & 5 \\
\hline wirkt & 25 & 18 & 23 & 25 & 10 & 28 & 13 & 10 & 12 & 16 & 180 & 8,505 & 1530,9 \\
\hline \multirow[t]{2}{*}{ wirken } & 13 & 11 & 10 & 15 & 5 & 14 & 9 & 8 & 5 & 5 & 95 & 6,046 & 574,37 \\
\hline & 40 & 30 & 33 & 42 & 16 & 42 & 22 & 19 & 17 & 22 & 283 & & 2113,27 \\
\hline Zuwachs & 40 & 70 & 103 & 145 & 161 & 203 & 225 & 244 & 261 & 283 & & & \\
\hline
\end{tabular}

Abbildung 5: Belegzahlen für die Konstruktion der Askription mit wirken im Kernkorpus des DWDS im Präsens Indikativ, differenziert nach Art des annotierten QUAL (VVPP/ADJD), Kommunikationsbereich (BE,GE,WI,ZE), Dekade

(des 20. Jh.) und morphologischer Form des finiten Verbs

Ein erstes grobes Ergebnis dieser Abfragen schließt Abbildung 5 auf, die wertvolle Hinweise liefert auf die Verteilung der Belege im Korpus. Die Konstruktion, die wir zu beschreiben suchen, scheint besonders häufig vertreten zu sein im Kommunikationsbereich „Gebrauchsliteratur“ (GE), während die Konstruktion fast nicht gebraucht wird in ,belletristischen Texten“ (BE). Interessant sind die Befunde für die „Zeitungssprache“ (ZE) hier gehen die Belege von einem niedrigen Niveau kommend in der vierten Dekade (das sind die Jahre 1931-1940) fast ganz zurück und bleiben bis ans Ende des Jahrhunderts auf diesem Stand. In Texten der Domäne „Wissenschaft“ (WI) sind Konstruktionen mit wirken und (deverbalem) Adjektiv auf mittlerem Niveau durchgängig nachweisbar. Diese Verteilung verstärkt sich noch einmal ganz erheblich, wenn man die Belegfaktoren mit ins Spiel bringt, deren Relevanz für die Einschätzung der Belegzahlen und tatsächlichen Verteilung im Korpus im Zusammenhang mit Abbildung 4 diskutiert wurde - die interpolierten Ergebnisse sind in der letzten, nicht farbig unterlegten Spalte in Abbildung 5 aufgeführt.

Um den Ausschnitt des Konstruktikons, der uns hier interessiert, nämlich die Konstruktionen der Askription mit wirken und deverbalem Adjektiv auf der Basis des Partizips II (vgl. dazu oben Abb. 3), präziser zu fassen, ist eine Aufschlüsselung der Ergebnisse nach Art der Qualitative notwendig, die zusammen mit wirken auftreten. Um diese zweifelsfrei 
zu bestimmen, ist eine Sichtung aller Belege notwendig, da - wie gesehen - die automatische Sortierung durch die Variation der Annotation in dieser Hinsicht leider nicht in Frage kommt (vgl. Abb. 6 und 7). In Abbildung 6 sind die Belege zusammengestellt, die durch die erste Analyse ermittelt werden konnten. Im Vergleich zu der Übersicht in Abbildung 5, für die die Daten noch nicht zum Teil manuell ausgewertet worden sind, tritt jetzt sehr deutlich und scharf heraus, wie die hier relevanten Instanzen der Konstruktion der Askription mit wirken und deverbalem Adjektiv (Partizip II) im Korpus verteilt zu sein scheinen (ich orientiere mich dabei immer noch an der Annotation der Daten). Durch Fettdruck sind sie in der Übersicht hervorgehoben und zwar in der einfachen Form [[wirken] + [VVPP]], der Koordination von Partizip I und II [[wirken] + [VVPS] + [VVPP]], Adjektiv und Partizip II [[wirken] + [VVPP] + [ADJD]] und schließlich allen adjektivischen Formen $[[$ wirken $]+[\mathrm{VVPS}]+[\mathrm{VVPP}]+[\mathrm{ADJD}]]$. Insgesamt stehen für eine Analyse 55 Belege zur Verfügung. Um den Stellenwert dieser Daten einschätzen zu können, wird nun die Interpolation anhand der Belegfaktoren (vgl. die Ausführungen zu Abb. 4) wichtig. Nimmt man die auf die auswertbaren Teile des Kernkorpus gerundeten Zahlen hinzu (vgl. Abb. 7), dann wird schnell klar, dass sich das Verhältnis noch deutlicher hin zur Verwendung von wirken mit VVPS und ADJD verschiebt und selbst bei Auswertung aller abgefragten Belege für Instanzen der Konstruktion der Askription mit wirken und deverbalem Adjektiv (Partizip II), deren Struktur sich darstellt wie in Abbildung 3 und die ich ins Zentrum meiner Überlegungen stelle, wahrscheinlich die absolute Zahl 140 nicht überschritten hätte.

$Z E$

wirken [VVPS]
wirken [VVPP]
wirken [ADJD]
wirken [VVPS] [VVPP]
wirken [VVPS] [ADJD]
wirken [VVPP] [ADJD]
wirken [VVPS] [VVPP] [ADJD]

SUMME NACH ART QUAL
D01 D02 D03 D04 D05 D06 D07 D08 D09 D10

$\begin{array}{rrrrrrrrrr}12 & 8 & 5 & 4 & 1 & 1 & 1 & 1 & 0 & 3 \\ 2 & 0 & 2 & 0 & 0 & 0 & 1 & 0 & 2 & 5 \\ 8 & 4 & 8 & 6 & 1 & 2 & 1 & 1 & 1 & 4 \\ 0 & 0 & 0 & 0 & 0 & 0 & 0 & 0 & 0 & 0 \\ 0 & 0 & 1 & 1 & 0 & 0 & 0 & 0 & 0 & 0 \\ 0 & 1 & 0 & 0 & 0 & 0 & 0 & 0 & 0 & 0 \\ 0 & 0 & 0 & 0 & 0 & 0 & 0 & 0 & 0 & 0\end{array}$

\section{D01 D02 D03 D04 D05 D06 D07 D08 D09 D10 GESAMT}

wirken [VVPS]
wirken [VVPP]
wirken [ADJD]
wirken [VVPS] [VVPP]
wirken [VVPS] [ADJD]
wirken [VVPP] [ADJD]
wirken [VVPS] [VVPP] [ADJD]

\begin{tabular}{|rrrrrrrrrr|r|}
\hline 62 & 28 & 38 & 34 & 18 & 36 & 16 & 10 & 11 & 15 & 268 \\
\hline 4 & 0 & 4 & 1 & 1 & 13 & 2 & 1 & 6 & 19 & $\mathbf{5 1}$ \\
21 & 21 & 24 & 28 & 10 & 28 & 12 & 16 & 10 & 17 & 187 \\
\hline 0 & 0 & 0 & 1 & 0 & 0 & 0 & 0 & 0 & 0 & 1 \\
\hline 1 & 0 & 3 & 2 & 2 & 0 & 1 & 0 & 1 & 0 & 10 \\
\hline 0 & 1 & 0 & 0 & 1 & 0 & 0 & 0 & 0 & 0 & 2 \\
0 & 1 & 0 & 0 & 0 & 0 & 0 & 0 & 0 & 0 & $\mathbf{1}$ \\
\hline
\end{tabular}

Abbildung 6: Nicht interpolierte Belegzahlen für die Konstruktion der Askription mit wirken im Kernkorpus nach Art des Qualitativs und Dekade 
ZE

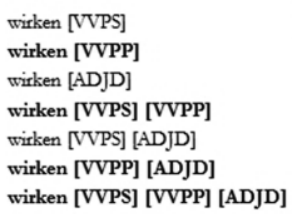

$\begin{array}{llllllllll}\text { D01 D02 D03 D04 D05 D06 D07 } & \text { D08 } & \text { D } & \text { D }\end{array}$

\begin{tabular}{|c|c|c|c|c|c|c|c|c|c|}
\hline 34,9 & 25,1 & 10,3 & 14,1 & 8,5 & 6,0 & 8,5 & 1,4 & 0,0 & 19,1 \\
\hline 4,1 & 0,0 & 2,9 & 0,0 & 0,0 & 0,0 & 8,5 & 0,0 & 3,5 & 15,5 \\
\hline 65,6 & 31,6 & 63,1 & 48,6 & 6,0 & 14,6 & 8,5 & 6,0 & 8,5 & 34,0 \\
\hline 0,0 & 0,0 & 0,0 & 0,0 & 0,0 & 0,0 & 0,0 & 0,0 & 0,0 & 0,0 \\
\hline 0,0 & 0,0 & 8,5 & 6,0 & 0,0 & 0,0 & 0,0 & 0,0 & 0,0 & 0,0 \\
\hline 0,0 & 2,1 & 0,0 & 0,0 & 0,0 & 0,0 & 0,0 & 0,0 & 0,0 & 0,0 \\
\hline 0,0 & 0,0 & 0,0 & 0,0 & 0,0 & 0,0 & 0,0 & 0,0 & 0,0 & 0,0 \\
\hline
\end{tabular}

wirken [VVPS]
wirken [VVPP]
wirken [ADJD]
wirken [VVPS] [VVPP]
wirken [VVPS] [ADJD]
wirken [VVPP] [ADJD]
wirken [VVPS] [VVPP] [ADJD]

$\begin{array}{llllllllll}\text { D01 D02 D03 } & \text { D04 } & \text { D05 } & \text { D06 D07 } & \text { D08 } & \text { D09 } & \text { D10 } & \text { GESAMT }\end{array}$

\begin{tabular}{|c|c|c|c|c|c|c|c|c|c|c|}
\hline 209,4 & 98,2 & 107,8 & 132,7 & 50,9 & 139,6 & 75,9 & 38,7 & 55,8 & 44,1 & 953,2 \\
\hline 7,0 & 0,0 & 6,4 & 1,4 & 1,4 & 30,4 & 10,0 & 2,1 & 11,1 & 49,2 & 119,0 \\
\hline 158,7 & 153,9 & 186,9 & 201,0 & 67,7 & 218,5 & 87,3 & 116,4 & 80,1 & 127,2 & 1397,7 \\
\hline 0,0 & 0,0 & 0,0 & 2,1 & 0,0 & 0,0 & 0,0 & 0,0 & 0,0 & 0,0 & 2,1 \\
\hline 6,0 & 0,0 & 23,1 & 12,1 & 17,0 & 0,0 & 8,5 & 0,0 & 6,0 & 0,0 & 72,8 \\
\hline 0,0 & 2,1 & 0,0 & 0,0 & 8,5 & 0,0 & 0,0 & 0,0 & 0,0 & 0,0 & 10,6 \\
\hline 0,0 & 6,0 & 0,0 & 0,0 & 0,0 & 0,0 & 0,0 & 0,0 & 0,0 & 0,0 & 6,1 \\
\hline
\end{tabular}

Abbildung 7: Interpolierte und gerundete Belegzahlen (Bezugsgröße ist der auswertbare Teil des Korpus) für die Konstruktion der Askription mit wirken im Kernkorpus differenziert nach Art des Qualitativs und Dekade

Leider stellen sich quantitative Vergleichsuntersuchungen (und damit der Ausweis statistischer Signifikanz) bisher als schwierig dar, da kein anderes deutschsprachiges Korpus zum einen hinsichtlich historischer Tiefe und zum anderen in Bezug auf unterschiedliche Kommunikationsbereiche geschichtet ist. Weiter hätte jede interpretative Entscheidung, wie anhand der nun zu diskutierenden Belege sofort sichtbar werden wird, erheblichen Einfluss auf die Einschätzung und Errechnung statistischer Signifikanz. Denn nicht alle nun in der Übersicht aufgeführten Daten erfüllen die Kriterien, die für die Beschreibung der Konstruktion der Askription mit wirken und deverbalem Adjektiv (Partizip II) aufgestellt worden sind, oder erweisen sich bei genauer Analyse, die sich der ersten manuellen Sortierung anschließt, als solche. Ich werde mich im Folgenden auf 20 exemplarische Belege (aus den genannten 55) konzentrieren, um die Konstruktion der Askription mit wirken und deverbalem Adjektiv (Part II) modellieren zu können. Sie werden - da sie hier eine Einheit bilden - separat gezählt (K für Korpus). Erscheinungsjahr, Kommunikationsbereich und Quelle sind angegeben. Die über den Suchstring aufgerufenen Einheiten (also z. B. in (K1) ÜBERLADEN und WIRKEN) werden mit Majuskeln hervorgehoben gemäß der Annotation im Korpus.

K1 | GE | 1901 | Doch sollte man hier ruhige Linien festhalten und zuviel Schmuck oder Verschnörkeleien vermeiden, die leicht ÜBERLADEN WIRKEN und nicht zum Charakter der Bücherei, die doch in der Hauptsache ernste oder doch gehaltvollere Bücher enthalten wird, paßt. || Baudissin, Wolf von / Baudissin, Eva von, Spemanns goldenes Buch der Sitte, Berlin, Stuttgart: Spemann 1901, S. 1958.

K2 | ZE | 1904 | Heymanns ist einer der wenigen Maler, die den Impressionismus selbständig verarbeitet haben, bei dem diese Malweise selbstverständlich und nicht ANGENOMMEN WIRKT. || Stahl, Fritz, Aus den Berliner Kunstsalons, in: Berliner Tageblatt (Morgen-Ausgabe) 03.03.1904, S. 2. 
K3 | GE | 1929 | Doch manche anderen Szenen erscheinen angesichts des interessanten und bedeutenden Themas allzu "neckisch" und WIRKEN allzu genrehaft ZURECHTGESTUTZT. | | Brenner, Max, Kulturfilme, in: Welt und Wissen 18 (1929) Nr. 1, S. 112-118.

K4 | WI | 1944 | Disharmonisch sind auch die Farben und das Verhältnis von Menschen und Raum - trostlos und wie kulissenhaft AUFGEBAUT WIRKEN die Zimmerwände. || Beenken, Hermann, Das Neunzehnte Jahrhundert in der deutschen Kunst, München: Bruckmann 1944, S. 321.

K5 | GE | 1955 | Der ganze Raum WIRKT "GESCHLOSSEN", während die unrubige Tapete ibn "auflöst". || Weber, Annemarie (Hg.), Die Hygiene der Schulbank, Wiesbaden: Falken [GS] Verl. 1955, S. 258.

K6 | GE | 1957 | Der Erfolg zeigt sich im Gesicht. Es WIRKT AUSGERUHT, GEPFLEGT, man sieht ihm den Arbeitstrubel der vorangegangenen Stunden nicht an. || Smolka, Karl, Gutes Benehmen von A - Z, Berlin: Verl. Neues Leben 1957, S. 14808.

K7 | BE | 1960 | Lieber Gott, vergib mir, wenn ich dich schon mal graue Mieze genannt habe, aber du WIRKST eben oft wahnsinnig VERSPIELT. || Walser, Martin, Halbzeit, Frankfurt a.M.: Suhrkamp 1960, S. 243.

K8 | GE | 1960 | Alle Seiten dieser Dichtung standen bei Fürnberg in Übereinstimmung, Privates verschmolz in Öffentlichem, Gesetzmäßigkeit schlug sich im Gedicht nieder, das wie intim DAHINGESPROCHEN WIRKT, scheinbar nur ihm zugehörig. || Wolf, Gerhard, Louis Fürnberg, in: Helmut Kaiser (Hg.), Die Dichter des sozialistischen Humanismus, München: Dobbeck 1960, S. 97-106.

K9 | WI | 1996| Der Exozytose ENTGEGENGESETZT WIRKT die Endozytose. || Zimmermann, Herbert, Molekulare Funktionsträger der Nervenzelle, in: Josef Dudel / Randolf Menzel / Robert F. Schmidt (Hg.), Neurowissenschaft, Berlin: Springer 1996, S. 33-61.

K10 | ZE | 1970 | Die Erklärung WIRKT WEIT HERGEHOLT und MANIERIERT. || o.A., Zeitmosaik, in: DIE ZEIT 13.03.1970, S. 20.

K11 | ZE | 1982 | Seine Munterkeit WIRKT GEZWUNGEN, seine Jovialität AUFGESETZT. || o.A., "Ändern Sie nie die Koalition", in: Der Spiegel 11.10.1982, S. 24-25.

K12 | ZE | 1989 | In den bisherigen Hauptsiedlungsgebieten veröden ganze Landstriche. Im türkischen Viertel der Stadt Schumen WIRKEN Straßenzüge wie AUSGESTORBEN. || o.A., Fortgang des Bevölkerungsexodus [21.08.89], in: Archiv der Gegenwart 59 (1989), S. 33684.

K13 | WI | 1994 Auf diesen Bildern WIRKEN die Arbeiter GENORMT || o.A., V, in: Harald Olbrich (Hg.), Lexikon der Kunst Band 1: Stae [GS] Z, Leipzig: Seemann 1994, S. 37169.

K 14 | ZE | 1997 | In seinem neuen, dunklen An₹ug WIRKT der Angeklagte GEHEMMT [...]. || Gernot Kramper, Party mit 170 PS, in: DIE ZEIT 13.06.1997, S. 69.

K15 | BE | 1999 | Plötzlich WIRKT der Mann sehr ERLEICHTERT, deutet ein Lächeln an und murmelt: "Na, dann wünsche ich dir noch einen schönen Abend [...]“. || Dückers, Tanja, Spielzone, Berlin: Aufbau-Verl. 1999, S. 32.

K16 | GE | 1999 | Paradoxerweise ist Nietzsche vielleicht am interessantesten als hellsichtiger Kritiker des Zeitgeistes der Dekadenz vor dem Ersten Weltkrieg. Er hat selbst etwas von einem Dekadenten, mixt wie ein Dandy Leben und Stil, WIRKT exaltiert (DURCHGEDREHT) und hysterisch, fühlt sich als Künstler und wird schließlich wahnsinnig, so daß er seine Briefe nur noch mit " Dionysos " oder " der Gekreuzigte " unterschreibt. || Schwanitz, Dietrich, Bildung, Frankfurt a.M.: Eichborn 1999, S. 344. 
K17 | GE | 1915 | Hochrot und Karminrot WIRKEN bekanntlich, nebeneinander STEHEND, WESENTLICH verändert. Sie ergänzen sich nicht, im Gegenteil, sie bekämpfen sich und geben zusammen keine Wirkung. || Hennig, Paul, Ueber Farbenwahl im Buchdruck, in: Mitteilungen des Vereins Deutscher Reklamefachleute (1915) Nr. 1, S. 21 [GS]22.

K18 | GE | 1935 | [Ein] scheinbar lustiges "Larà, larà" WIRKT wie atemlos, ABGERISSEN, lauernd | | Schuhmann, Otto, Meyers Opernbuch, Leipzig: Bibliograph. Inst. 1935, S. 281.

K19 | GE | 1942 | Das wachsende Leben muß auch ernährt werden. Das Unterhautfettgewebe der Frau ist gut entwickelt, wie überhaupt der weibliche Körper viel Fett enthält (25\% gegen $10 \%$ im männlichen Körper). Er WIRKT dadurch in allen seinen Teilen WEICH und gerundet. Diesen Gegebenheiten müssen sich Sport und Gymnastik unterordnen. || Wiehle, Hermann / Harm, Marie, Lebenskunde für Mittelschulen - Klasse 5 für Mädchen, Halle [u. a.]: Schroedel [u. a.] 1942, S. 76.

K20 | ZE | 1920 | Wie ganze Betrachtungsweisen sachte untergehen, nicht mehr wirksam sind, wie wir geliebte Bücher nicht mehr lesen können, weil die Voraussetzungen nicht mehr stimmen, und wie Maupassant hölzern und tot und GESTRECKT WIRKT, weil alles anders geworden ist unterdessen. || Tucholsky, Kurt, Leerlauf, in: Die Weltbühne 30.09.1920, S. 373.

Zunächst möchte ich die Aufmerksamkeit lenken auf den Beleg (K9): Der Exozytose entgegengesetzt wirkt die Endozytose. Der Beleg wurde automatisch den hier zu untersuchenden Belegen zugeordnet, da in die Konstruktion ein deverbales Adjektiv der Form eines Partizips II und wirken eingebettet ist; auch die erste manuelle Selektion, die sich an der Markierung der Abfrage orientierte (hier die Hervorhebung des Partizips in Majuskeln) und in den Abbildungen 6 und 7 repräsentiert ist, folgte dieser Zuordnung. Bei der genauen Analyse, die wir jetzt vornehmen, muss diese Zuordnung jedoch korrigiert werden - das ist ein erster Hinweis darauf, dass die postulierte scharfe Trennung zwischen zwei Subtypen der Konstruktion der Askription (vgl. Abb. 2 und 3) mit wirken allein auf der Basis der Besetzung der Argumenstrukturrolle des QUAL nicht möglich ist. Das hat zwei Gründe: Zum einen dürfte die Einschätzung, ob entgegensetžt im Beleg als Adjektiv oder als deverbales Adjektiv auf der Basis eines Partizip II zu verstehen sei, nicht ohne Zusatzannahmen möglich sein. Zum anderen stellt die Einstufung des Agentivitätsgrades der Endosytose unser Verständnis von Agentivität, auch wenn man einen mehrdimensionalen Agensbegriff zu Grunde legt, auf eine harte Probe. Das hat zur Folge, dass für den Beleg drei Lesarten durchgespielt werden können: (1) ,Als Endozytose (=Stoffwechselvorgang) wird ein Vorgang bezeichnet, bei dem zellfremde Elemente im Gegensatz zur Exozytose in eine Zelle aufgenommen werden', (2) ,Die Endozytose ist ein Prozess. Dieser hat die Eigenschaft, in entgegengesetzter Richtung der Exozytose zu wirken' (Konstruktion der Askription mit direkter Relation zwischen Verb- und Konstruktionsbedeutung, vgl. oben Abb. 2) und (3) markiert eine Sprecherbehauptung hinsichtlich der Faktizität mit ,Es scheint so, als ob die Endozytose der Exozytose entgegenwirke (Konstruktion der Askription mit modaler Relation zwischen Verb- und Konstruktionsbedeutung, vgl. oben Abb. 3). Die Lesarten (2) und (3) sind unter Berücksichtigung des Kontextes recht unwahrscheinlich, wenn nicht gar ungrammatisch. Entgegengesetît wird als Adjektiv in der Funktion eines Adverbials aufzufassen sein und damit ist die Lesart (1) die plausible.

Die Belege (K1), (K3), (K5), (K6) und (K8), (K11-15) legen wie zunächst (K9) der Form nach nahe, dass es sich hierbei um Realisierungen der Konstruktion der Askription mit wirken mit modaler Relation zwischen Verb- und Konstruktionsbedeutung (vgl. oben Abb. 3) handelt. Bei genauer Interpretation erweisen sie sich auch als solche: 


\begin{tabular}{|l|l|l|l|}
\hline Beleg & SOB & V & QUAL \\
\hline K1 & die [Verschnörkeleien] & wirken & (leicht) überladen \\
\hline K3 & manche anderen Szenen & wirken & (genrehaft) zurechtgestutzt \\
\hline K5 & der ganze Raum & wirkt & "geschlossen “ \\
\hline K6 & es [das Gesicht & wirkt & ausgerubt, gepflegt \\
\hline K8 & das [Gedicht & wirkt & (intim) dabingesprochen \\
\hline K11 & seine Munterkeit, Jovialität & wirkt & gezwungen; aufgesetzt \\
\hline K12 & Straßenzüge & wirken & ausgestorben \\
\hline K13 & die Arbeiter & wirken & genormt \\
\hline K14 & der Angeklagte & wirkt & gehemmt \\
\hline K15 & der Mann & wirkt & erleichtert \\
\hline
\end{tabular}

Tabelle 1: Realisierungen der Konstruktionen der Askription mit wirken und deverbalem Adjektiv (Partizip II) mit modaler Relation zwischen Verb- und Konstruktionsbedeutung und Angabe der Kommunikationsdomäne

Anders als in den Belegen, in denen Adjektiv und Partizip II separat ausgewiesen sind, besetzen die Adjektive in (K1), (K3) und (K8) nicht den Slot des Qualitativs, sondern beziehen sich modifizierend entweder auf den Qualitativ oder übernehmen die Funktion eines Adverbials. Der Qualitativ ist in allen Belegen durch ein deverbales Adjektiv (Partizip II) besetzt. Eine weiterführende Klassifizierung der Elemente, die in den Slot des Spezifizierten Objektes (SOB) und des Qualitativs (QUAL) eintreten, ist jedoch nicht möglich: Das SOB kann belebt sein oder nicht, menschlich oder nicht, abstrakt oder konkret; das deverbale Adjektiv in Form eines Partizips II kann gebildet sein auf der Basis perfektiver (telischer), transitiver Verben (überladen, zurechtstutzen, scbließen, [sich] ausruben, [sich] pflegen, aufsetzen, aussterben, ?normen, erleichtern), oder imperfektiver (atelischer), transitiver Verben (zpingen, dabinsprechen, ?normen, hemmen). Durch die Bildung des Partizips II wird das Potential der Aktionsart der Verben jedoch vereindeutigt hin zu einer perfektiven (telischen) Lesart, die dann in die Bedeutung des deverbalen Adjektivs eingeht: Als „Spiegel des Perfekts“, so wurde Welke (2005: 238) bereits zitiert, lassen sich die Partizipien II perfektiver Verben auffassen; imperfektiven Verben wird diese Konstruktionsbedeutung gewissermaBen eingeschrieben (vgl. Welke 2005: 196 und besonders 238-240). Man kann auf der Basis dieser ersten Ergebnisse also knapp zusammenfassen: Zur semantischen Eigenschaft des Qualitativs gehört es, die Lesart der Konstruktion der Askription mit wirken ( $\approx$ modal) für die beschriebenen Belege zu begünstigen. In den besprochenen Fällen wird die Perfektivität durch die Bildung des Partizips II erreicht. Besonders herauszuheben ist in diesem Zusammenhang der Beleg (K3), in dem im linksperipheren nebengeordneten Matrixsatz das Verb erscheinen zusammen mit dem Adjektiv neckisch in einer Realisierung derselben Konstruktion eingebettet ist und unsere Interpretation stützt.

Weiter sind konventionalisierte kollokative Bezüge zwischen den Elementen, die als SOB und QUAL eingebettet sind, Voraussetzung, um sie einander zuzuordnen. Wie fein diese Merkmale im Gebrauch aufeinander abgestimmt werden müssen und daher auch die Analyse erschweren, wird der zweite Teil der Belege illustrieren, die nun im Mittelpunkt des Interesses stehen. 


\begin{tabular}{|c|c|c|c|}
\hline Beleg & SOB & $\mathrm{V}$ & QUAL \\
\hline K2 & diese Malweise & wirkt & selbstverständlich; (nicht) angenommen \\
\hline K4 & die Zimmerwände & wirken & trostlos; (kulissenhaft) aufgebaut \\
\hline K10 & die Erklärung & wirkt & (weit) hergebolt, manieriert \\
\hline K16 & er [Nietzsche] & wirkt & exaltiert; durchgedreht; hysterisch \\
\hline K20 & {$[e s]$} & wirkt & bölzern; tot; gestreckt \\
\hline
\end{tabular}

Tabelle 2: Realisierungen der Konstruktionen der Askription mit wirken, deverbalem Adjektiv (Partizip II) und Adjektiv mit modaler Relation zwischen Verb- und Konstruktionsbedeutung und Angabe der Kommunikationsdomäne

In allen Belegen wird die Konstruktion mit wirken sowohl mit einem und zum Teil mit mehreren Adjektiven (selbstverständlich, trostlos, manieriert, exaltiert, hysterisch, bölzern und tot) sowie meist mit einem deverbalen Adjektiv auf Basis eines Partizips II (angenommen, aufgebaut, hergeholt, durchgedreht, gestrecket) realisiert, obwohl die Belege (K4), (K10) und (K16) weiterer Erläuterung bedürfen. In Beleg (K4) ist die Zuordnung zwischen den verwendeten Adjektiven trostlos, kulissenhaft und dem Partizip II aufgebaut nicht eindeutig bestimmbar - ich habe mich hier für die plausibelste Lesart entschieden: trostlos ist als ein Qualitativ eingebettet und (kulissenhaft) aufgebaut als zweiter Qualitativ, verbunden sind beide durch die Konjunktion und. Die Lesart , die Zimmerwände können trostlos aufgebaut und kulissenhaft aufgebaut scheinen', liegt dagegen nicht nahe, kann aber auch nicht ausgeklammert werden. (K16) ist ein besonderer Fall, da hier eine Realisierung der Konstruktion nur mittels Adjektiven im Slot des Qualitativs realisiert wird, das deverbale Adjektiv auf der Basis des Partizips II durchgedreht kommt nur als nähere Erläuterung eines Adjektivs (exaltiert) ins Spiel und ist demzufolge auch eingeklammert. (K10) schließlich stellt das vorgeschlagene Modell auf die Probe, denn die Verbindung zwischen Adjektiv und Partizip II weit hergeholt hat phraseologische Qualität - ,etw. von weit her holen' wird metaphorisch gebraucht und bildet eine stabile und konventionalisierte Einheit, die lexikalisch teilspezifiziert ist und eigenen konstruktionalen Status beanspruchen darf. Insofern wäre die Einklammerung von weit in der Übersicht zu diskutieren. Mit Ausnahme von (K16) sind in der Belegreihe alle SOB durch NP im Nominativ besetzt, die keine Merkmale von Agentivität tragen - mit anderen Worten, eine agentive Lesart oder eine Vorgangslesart scheidet für diese Fälle aus. (K16) ist für unsere Analyse ein interessanter Fall: Auch wenn es sehr unüblich wäre, der Nominalphrase Nietəsche in Nietzsche wirkt exaltiert und hysterisch die Rolle des Agens zuzuweisen, so ist die Lesart nicht ausgeschlossen; erst durch das verwendete Partizip II durchgedreht wird die nonagentive Lesart wahrscheinlicher und die Konstruktion erweist sich als eine der Askription mit wirken mit modaler Relation zwischen Verb- und Konstruktionsbedeutung: ,Nietzsche erscheint exaltiert (durchgedreht) und hysterisch'.

Auch in der zweiten Belegreihe bilden perfektive Verben die Basis für die gebildeten Partizipien, die als deverbale Adjektive in die Konstruktion eingebettet sind. Sie stellen, wie in (K16) gesehen, die Lesart der Konstruktion der Askription sicher, auch wenn die als SOB eingebettete Nominalphrase die Merkmale belebt/menschlich trägt. Die qualifizierenden Adjektive, die in den Belegen verwendet werden, können hier nicht im Detail semantisch beschrieben werden, auch weil nicht auf eine semantische Klassifikation von Adjektiven zurückgegriffen werden kann. ${ }^{40}$ Ihnen allen ist gemeinsam, dass sie - wiederum bis auf exaltiert und bysterisch - keine semantischen Potentiale haben, die auch im Kontext

40 Vgl. die Diskussion der Klasse exemplarisch bei Eisenberg (2006 I: 177-184) und zur Rolle in satzwertigen Ausdrücken besonders (2006 II: 223-230 [Adverb] sowie 237-246 [Attribut]). 
ihrer Kollokationen auf eine Dynamik (wie etwa bspw. langsam, schnell, rastlos, erfahrbar etc.) verwiesen. Sie werden als qualifizierende Adjektive eingeordnet, die „einer Person oder Sache eine Eigenschaft zuordnen“ (Duden-Grammatik 2009: 358) und damit die Rolle des QUAL von ihrer Bedeutung her ideal besetzen. Funktional können die meisten nicht adverbial, sondern nur attributiv und - wie hier - prädikativ gebraucht werden. Eine Ausnahme bilden die bereits für (K16) problematisierten Beispiele exaltiert und hysterisch. Damit sind die in die Konstruktion eingebetteten Adjektive den hinsichtlich ihrer Perfektivität markierten Partizipien II strukturell vergleichbar: Sie bezeichnen aus semantischer Perspektive eine Eigenschaft einer „Person, einer Sache oder eines Sachverhaltes, nicht aber die Art und Weise eines Ereignisses oder Zustandes“ (Duden-Grammatik 2009: 358), können aus funktionaler Perspektive nicht adverbial, sondern nur attributiv und prädikativ gebraucht werden.

\begin{tabular}{|l|l|l|l|}
\hline Beleg & SOB & V & QUAL \\
\hline K7 & $d u[$ lieber Gott & wirkst & (oft wahnsinnig) verspielt \\
\hline K17 & Hochrot und Karminrot & wirken & $\begin{array}{l}\text { (nebeneinander) stehend; (bekannt- } \\
\text { lich wesentlich) verändert }\end{array}$ \\
\hline K18 & $\begin{array}{l}\text { ein scheinbar lustiges } \\
\text { „Lară, larä“ }\end{array}$ & wirkt & atemlos; abgerissen; lauernd \\
\hline K19 & $\begin{array}{l}\text { er [der weibliche Körper] } \\
\text { wirkt }\end{array}$ & weich; gerundet \\
\hline
\end{tabular}

Tabelle 3: Realisierungen der Konstruktionen der Askription mit wirken, deverbalem Adjektiv (Part II) und/oder Adjektiv mit modaler Relation zwischen Verb- und Konstruktionsbedeutung und Angabe der Kommunikationsdomäne

Die letzten vier Fälle in der Belegreihe scheinen Spezialfälle zu sein, die teils aufgrund der Annotation des Korpus, teils wegen der Kriterien der ersten manuellen Sortierung einer solchen Kategorie zugeordnet werden könnten, aber nicht sollen: Konstruktionsgrammatisch sind diese Belege, die immer die Grenzen der Modellbildung aufzuzeigen in der Lage sind, gerade zur Erklärung und Plausibilisierung von Konstruktionen und ihren Strukturen sowie deren Einbettung in einem Konstruktikon herangezogen werden, um den Erklärungsansatz zu plausibilisieren. Blicken wir zunächst auf den Beleg (K19). Obwohl hier die automatische und die manuelle Sortierung ein Adjektiv (weich) und ein Partizip II (gerundet) verzeichnen, ist der Fall nicht unproblematisch: Das Verb, auf das sich das Partizip II gerundet bezieht, ist runden. Gemeint ist damit hier so etwas wie ,etw. eine rundliche Form geben, etwas rund machen'; die perfektive Qualität des Verbs wird durch die Bildung des Partizips II noch verstärkt. Gerundet kann jedoch auch als Adjektiv eingestuft werden, da die Bedeutung des Verbs, das dem Partizip II zu Grunde liegt, hier nur in einem übertragenen Sinne gelten kann. Das würde in unserem Fall aber die Lesart nicht verändern, hätte nur direkten Einfluss auf die Zählung der Ergebnisse: Die im SOB eingebettete NP hat hier nur schwache Merkmale der Agentivität, wirken kann hier also schlecht in seiner Vollverbsematik verstanden werden. Die Adjektive weich und gerundet wiederum können aufgrund ihres semantischen Potentials funktional nicht als Adverbiale verwendet werden. Plausibel hingegen ist die Realisierung der Konstruktion der Askription mit wirken $(\approx$ modal) mit zwei Adjektiven, die als QUAL eingebettet sind. Was sich jedoch an diesem Beispiel (auch noch einmal in Bezug zu K16) besonders deutlich zeigen lässt, ist, dass einer der Qualitative sich durch das semantische Merkmal der Perfektivität bzw. geringes Dynamikpotential auszeichnen muss, damit diese Lesart wahrscheinlich wird, die durch einen abnehmenden Agentivitätsgrad des SOB begünstigt wird. Wie diese Merkmale ineinandergreifen, lässt sich sehr gut am Beleg (K7) illustrieren. Wie in (K19) wird verspielt durch den 
Zugriff auf die Korpusdaten als Partizip II identifiziert (als Partizip zu etw. verspielen), was aber nicht plausibel erscheint. Verspielt ist Adjektiv und wird hier durch zwei weitere Adjektive in einer komplexeren Gruppe modifiziert (oft wabnsinnig). Der Beleg ist hinsichtlich seiner Lesarten ambig, er kann agentiv (verspielt ist dann adverbiales Adjektiv) oder nonagentiv als Subtyp der Konstruktion der Askription mit wirken ( $\approx$ modal) interpretiert werden - letztere Lesart ist eher wahrscheinlich, was wie im Beleg (K16) trotz der zweifellos unbestreitbaren Agentivität des lieben Gottes in der Form und im semantischen Potential des Qualitativs begründet sein dürfte. (K17) erscheint auf den ersten Blick als recht komplex, zumindest in Bezug auf die automatische Codierung der Qualitative. Bei genauerem Hinsehen erweist sich (nebeneinander) stehend als Parenthese und der Aussagezusammenhang, der hier interessiert, ist durch das modifizierte Partizip II verändert aufgerufen - dieses dominiert in Bezug auf die zu wählende Lesart den Einschub. Das allerdings ist anders in Beleg (K18): [Ein] scheinbar lustiges „Larà, larä“ wirkt wie atemlos, abgerissen, lauernd. Ein Adjektiv, ein Partizip II und ein Partizip I werden als Qualitative nebengeordnet in einer Konstruktion der Askription mit wirken - und der Subtyp mit modaler Relation zwischen Verbbedeutung und Konstruktionsbedeutung wird als Lesart erzwungen. Aus der Analyse der Belege lassen sich hierfür als Gründe anführen, dass das gesungene Larà, larà (SOB) keine Agentivitätsmerkmale aufweist, atemlos den Adjektiven zuzuordnen ist, die das Potential haben, eine Eigenschaft einer Person oder Sache zu beschreiben, aber nicht die Art und Weise eines Ereignisses, abgerissen als Partizip II von abreißen durch ein hohes Maß an Perfektivität ausgezeichnet ist und lauernd als Partizip I wie atemlos zwar dynamisches Potential andeutet, aber nicht ausdrückt und weiter tendenziell als Kollokat eher selten zu einem gesungenen Lied in Erscheinung treten wird. Entschließt man sich weiter dazu, lauernd den Status eines deverbalen Adjektivs zuzuweisen, den es in einem Grammatikalisierungsprozess erreicht hat, dann ist die Lage noch eindeutiger - einen Test, ob und unter welchen Bedingungen lauernd adverbial gebraucht werden kann, müsste man in einer Korpusanalyse noch erbringen. ${ }^{41}$

\section{Die Konstruktion der Askription mit wirken im Konstruktikon}

Auch der letzte Beleg, den ich hier besprechen möchte, stammt wie (K7) von Martin Walser, der - traut man den Korpusdaten - eine Vorliebe für die von uns untersuchte Konstruktion zu haben scheint. Eine besondere Realisierung, die aufgrund des Zuschnitts dieses Beitrages (hier standen Belege im Präsens Indikativ im Mittelpunkt) nicht Gegenstand der Analyse wurde, ist diese:

41 Die manuell zu bewertenden Belegzahlen halten sich dafür im DWDS wirklich im Rahmen: Die Abfrage @lauernd* $\& \& ! \$ \mathrm{p}=\mathrm{NN}$ (,lauernd nicht gefolgt von Nomen') gibt 38 Belege aus (davon sind 25 auswertbar). Will man die Auswahl nicht voreinschränken (@lauernd*), ruft man 401 Belege auf, von denen jedoch nur insgesamt 262 zur Analyse freigegeben sind (<http://www.dwds.de>, Stand: 04.03.2014). 


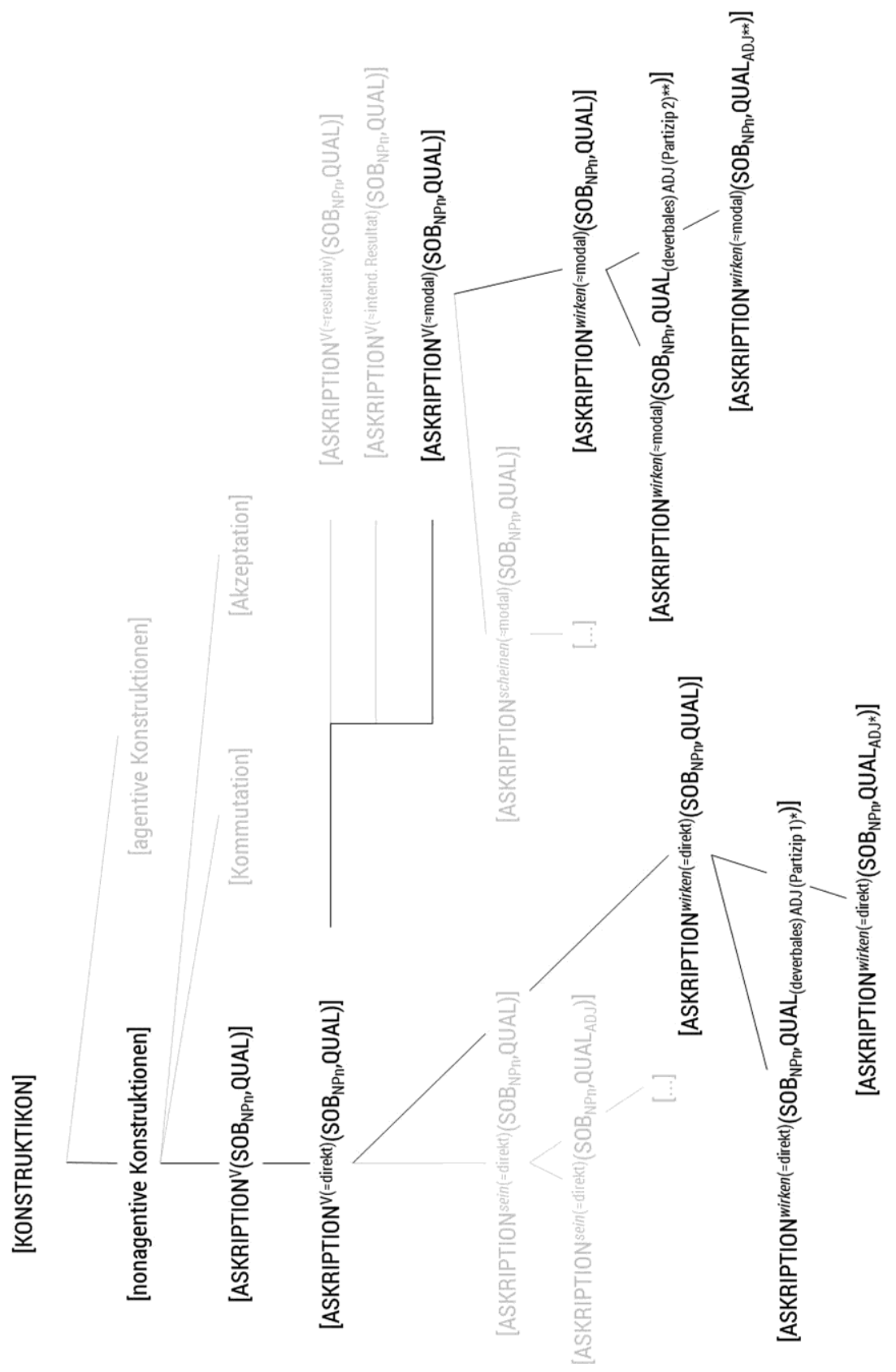

Abbildung 8: Die Konstruktionen der Askription mit wirken im Konstruktikon (Nicht thematisierte Knoten und Beziehungen sind ausgegraut.) 
(12) $1957 \mid \mathrm{BE} /$ Sie war schön und groß und wirkte gleichzeitig mächtig, voll gewachsen und doch zart und fast durchsichtig. || Walser, Martin, Ehen in Philippsburg, Frankfurt a. M.: Suhrkamp 1957, S. 51.

Zahlreiche Adjektive, auch eines auf der Basis des Partizips II, werden als Qualitative in die Konstruktion der Askription mit wirken ( $\approx$ modal) in $(12)$ eingebettet und zudem durch weitere Adjektive modifiziert: (gleichzeitig) mächtig, (voll) gewachsen, zart und durchsichtig. Alle Adjektive bestimmen die ,Eigenschaften einer Person oder Sache' näher und das Partizip II als Konstruktion lizenziert das Merkmal der Perfektivität für das eingebettete imperfektive Verb wachsen. Wie in Beleg (K3) tritt im nebengeordneten Matrixsatz eine weitere Realisierung der Konstruktion der Askription auf - die Verbbedeutung von sein interagiert anders als die von wirken mit (deverbalem) Adjektiv (Partizip II) direkt mit der Konstruktionsbedeutung, so dass an diesem Beispiel die markierte Faktizitätsbehauptung mit wirken durch den Sprecher deutlich hervortritt. Belege wie dieser sind besonders gut geeignet, die Struktur von Konstruktionen und ihre Beziehungen zueinander darzustellen. In Abbildung 8 werden die Konstruktionen und Konstruktionstypen bedeutungsseitig einander zugeordnet und als Ausschnitt des Konstruktikons präsentiert.

Gegenstand des Artikels waren die Konstruktionen der Askription mit wirken in zwei Varianten - die direkte Relation zwischen Verbbedeutung und Konstruktionsbedeutung war zunächst postuliert worden für die Einbettung von wirken und einem deverbalen Adjektiv der Form des Partizips I. Die Analyse der gebrauchsbasierten Daten ergab, dass auch Adjektive in die Konstruktion eingebettet werden können, die in satzwertigen Ausdrücken die Funktion eines Adverbials übernehmen, da sie die „Art und Weise eines Ereignisses oder Zustandes“ (Duden-Grammatik 2009: 358) bestimmen können, wie rastlos in (9a) Lewin wirkt rastlos (im Schaubild ADJ*). Da die Variablen für diese Verteilungsverhältnisse nach den Eingangsüberlegungen nicht zweifelsfrei bestimmt werden konnten, stand die Konstruktion der Askription mit wirken in modaler Relation zwischen Verb- und Konstruktionsbedeutung im Mittelpunkt des Beitrags. Hier werden (deverbale) Adjektive der Form des Partizips II und Adjektive eingebettet, die „nur die Eigenschaften einer Person, einer Sache oder eines Sachverhaltes“ (Duden-Grammatik 2009: 358) bestimmen, wie die Analyse zeigte (im Schaubild ADJ**). Eine Variable, deren Einfluss nach der Untersuchung relativiert werden muss, ist die der Agentivität des SOB. Grundsätzlich kann zwar gesagt werden, dass mit einem zunehmenden Agentivitätsgrad der Nominalphrase im Nominativ, die den Slot des SOB besetzt, auch eine agentive Lesart mehr und mehr begünstigt wird, aber diese wird insbesondere dann aufgerufen, wenn in die Konstruktion deverbale Adjektive auf der Basis eines Partizips I und die Adjektive, die auch adverbial verwendet werden können, eingebettet sind. Wie in (9a) Lewin wirkt rastlos kann dann mit letzter Sicherheit und ohne Kontextinterpretation keine Aussage darüber gemacht werden, ob hier eine nonagentive oder agentive Konstruktion realisiert wurde. (Deverbale) Adjektive auf der Basis eines Partizips II und Adjektive, die nur attributiv oder prädikativ gebraucht werden, hingegen stabilisieren die Lesart der nonagentiven Konstruktion der Askription mit wirken in modaler Relation zwischen Verb- und Konstruktionsbedeutung und blockieren eine agentive Lesart, was zum einen dem semantischen Potential der Adjektive und zum anderen dem Merkmal der Perfektivität zuzuschreiben ist, das die Konstruktion der Partizipien II auszeichnet.

So müssen wir hier - den vermutlich nicht so seltenen - Fall postulieren, dass wirken sowohl in Konstruktion der Askription mit direkter Relation als auch in modaler Relation 
zwischen Verb- und Konstruktionsbedeutung eingebettet sein kann. Konstruktionen mit modaler Relation erben ihre Eigenschaften von denen mit direkter Relation und sind durch diese motiviert (Goldberg 1995: 72) und stehen über Polysemie-Beziehungen (polysemy links) in Verbindung (vgl. dazu ausführlich Goldberg 1995: 74-97). Phänomene der multiplen Vererbung von Eigenschaften auf Konstruktionsebene werden unmittelbar am Verb wirken selbst sichtbar, sonst könnte dieses als Konstruktion niederer Ordnung nicht in verschiedene Konstruktionstypen auf anderen Ebenen eingebettet werden - beide Verwendungsweisen, die hier im Mittelpunkt standen, erben ihre Eigenschaften von wirken in agentiver Lesart über die metaphorische Erweiterung (metaphorical extension links, das gilt für die direkte Relation, vgl. Abb. 2) bzw. Polysemie-Beziehungen (polysemy links, das gilt für die modale Relation, vgl. Abb. 3), aber auch von scheinen hinsichtlich der Markierung der Faktizitätsbehauptung durch den Sprecher. In dieser Lesart stehen sie wiederum in Beziehungen zu Konstruktionen mit Modalverben, die hier nicht besprochen werden konnten. Dass die Verhältnisse - vor allem im letzten Punkt - nicht klarer sind, deutet auf einen Grammatikalisierungsprozess von wirken hin, den dieses durch die Einbettung in die Konstruktion der Askription mit direkter Relation zwischen Verb- und Konstruktionsbedeutung durchläuft, ohne dabei gleichzeitig das Potential zu verlieren, in agentive Konstruktionen eingebettet werden zu können. ${ }^{42}$ An anderer Stelle ist dieser Prozess begünstigt durch die Art der eingebetteten Qualitative abgeschlossen: Verdeutlichen kann man sich das an den Beispielen (K3) und (12), in welchen je zwei Realisierungen der Konstruktion der Askription entweder mit modaler Relation zwischen Konstruktions- und Verbbedeutung mit erscheinen und wirken vorliegen oder direkter Relation und modaler Relation mit sein und wirken.

Abbildung 8 zeigt nur einen Ausschnitt des Konstruktikons. Er zeigt die nonagentiven Konstruktionen und die Konstruktion der Askription als Knoten im Netzwerk auf einer höheren Abstraktionsebene. Der Ausschnitt ist vereinfacht dargestellt, da sich der Beitrag auf adjektivische Qualitative beschränkte. So wurden auch nur diese in die Strukturdarstellung mit aufgenommen, womit keinesfalls mitbehauptet sein soll, dass andere Arten an Qualitativen in die Konstruktion der Askription nicht eingebettet werden könnten. Wie weit hier das Spektrum reicht, das in gebrauchsbasierten Studien aber noch nicht vollständig rekonstruiert ist, kann man sich vergegenwärtigen an den verschiedenen Formen, die zusammen mit dem Verb sein in die Konstruktion eingebettet sein können: Die Einbettung von Nominalphrasen im Nominativ (Lewin ist Lehrer), am-Progressiven (Lewin ist am Abschreiben) oder erweiterten Infinitiven mit zu (Lewin ist zu tadeln) muss noch detailliert beschrieben werden, um eine Integration in den Ausschnitt de Konstruktikons, wie er sich jetzt in Abbildung 8 darstellt, plausibel zu begründen.

\section{Zusammenfassung und Ausblick}

Es gibt - wie auch die Beiträge in diesem Band zeigen - keine einheitliche Auffassung über die Art und Weise, wie das Konstruktikon zu verstehen sei, vielleicht ist das auch überhaupt nicht möglich oder nötig. Ein Vorschlag wäre, das Konstruktionsnetzwerk vor allem über das semantische Potential und die Perspektivierungsleistung von Konstruktionen zu

42 Vgl. zum Verhältnis von Grammatikalisierung und Konstruktionswandel Hilpert (2013). 
entwerfen. Mittels Aussagerahmen, Prädikationsrahmen, Prädikationsklassen und semantischer Rollen sowie der Überlegungen zur Struktur einer Konstruktion ist es möglich, die Knoten und Verbindungen zwischen Konstruktionen zunächst auf semantischer und dann auf formaler Ebene vorzunehmen und vor allem Konstruktionen unterschiedlichen Komplexitätsgrades zu berücksichtigen.

Ungeachtet einiger offener Punkte und Probleme konnte gezeigt werden, dass und wie wirken $\mathrm{u}$. a. in die Konstruktion der Askription eingebettet wird. Aus unterschiedlichen Verwendungsweisen sind Rückschlüsse möglich auf den Grammatikalisierungsstand des Verbs hinsichtlich unterschiedlicher Verwendungsweisen und Gebräuche, die wiederum eine Zuordnung zu unterschiedlichen Prädikatsklassen erlauben. Ich konzentrierte mich hier auf Fälle, in denen wirken der Prädikatsklasse ZUSTAND zugeordnet werden kann. Abhängig von der Art des eingebetteten Qualitativs wurden zwei Typen der Realisierung der Konstruktion entlang der Art der Relation zwischen Konstruktions- und Verbbedeutung (direkt/modal; vgl. Abb. 8 sowie die Erläuterungen in Abschnitt 5) bestimmt, was wirken zu einem interessanten Fall macht, wenn man über Konstruktionsnetzwerke nachdenkt - genau dies ließen die anderen eingangs diskutierten Darstellungen vermissen, die wirken nicht bzw. nur am Rande in dem hier dargestellten Kontext berücksichtigten. Die qualitative Korpusanalyse brachte schließlich entscheidende Hinweise auf die Bedingungen für die Einbettung des Verbs in die Konstruktion der Askription ans Licht.

Daneben konnte der Beitrag weitere Dinge aufzeigen, die sich auf den Umgang der Konstruktionsgrammatik mit gebrauchsbasierten Sprachdaten beziehen. (1) Um sprachliche Einheiten im Gebrauch analysieren zu können, sind zunächst die Funktionen, die einzelne sprachliche Einheiten in größeren sprachlichen Einheiten übernehmen (hier wurden vor allem die Adverbiale und der Prädikativ tragend) können, auszublenden. Funktionale Zusammenhänge sollen und können Erklärungen liefern, sind aber aus der strukturellen Beschreibung von Konstruktionen unbedingt auszuklammern. Diese sollen sich auf die Beschreibung von semantischen Eigenschaften (semantische Rollen) und formale Eigenschaften (z. B. Wortarten bzw. Phrasen) beschränken. (2) Gebrauchsbasierte Studien sind auf die Qualität annotierter Korpora angewiesen. Stellt man konstruktionsgrammatisch motivierte Fragen, ist dieser Annotation (im Moment noch) u. U. nicht zu trauen. Die Beurteilung, ob eine sprachliche Einheit - wie hier - als Adjektiv oder als Partizip eingestuft wird, verzerrt sämtliche Versuche, sprachliches Material automatisch zu quantifizieren bzw. erfordert manuelle Selektion und Interpretation in einem Maße, dass der Einsatz im Hinblick auf die erzielten Ergebnisse auch forschungspraktisch fraglich wird. Allerdings sind selbst die ersten ungenauen halbautomatisch erzielten Ergebnisse so genau, dass zumindest Aussagen hinsichtlich einer Tendenz der Sprachentwicklung und des Sprachgebrauchs möglich sind - aber (im Moment) nicht mehr. (3) Die Ungenauigkeiten bei der Annotation weisen auf ein tiefer liegendes Problem, das wiederum als ein Forschungsdesiderat $\mathrm{zu}$ identifizieren ist. Wortartklassifikationen, wie man sie üblicherweise verwendet, sind Kreuzklassifikationen, die mit formalen und semantischen Variablen arbeiten. Die Klasse der Adjektive ist in der Konsequenz so heterogen, dass die Bestimmung eines QUAL, so wie hier, ebenfalls auf formale und semantische bis funktionale Merkmale zurückgreifen muss. Ob es dafür in naher Zukunft eine befriedigende Lösung geben kann, ist fraglich - es käme aber auf den Versuch an. Ein erster Schritt wäre, das zeigte auch die Analyse, in der Annotation gänzlich auf funktionale Merkmale zu verzichten und nur noch formale Eigenschaften zu berücksichtigen. Geschlossen in Das ist ein geschlossenes Fenster wird 
dann nicht als attributiv gebrauchtes Adjektiv verzeichnet, sondern als (deverbales) Adjektiv auf der Basis eines Partizips II.

\section{Literatur}

Ackerman, Farrell \& Gert Webelhuth. 1998. A Theory of Predicates. Stanford: CSLI Publications.

Boas, Hans C. 2010. The Syntax-Lexicon Continuum in Construction Grammar: A Case Study of English Communication Verbs. In: Belgian Journal of Linguistics 24, 57-86.

Breindl, Eva. 2011. Art.: „Kopula“. In: Grammis 2.0 (IdS). Abrufbar unter: <http://goo.gl/3D43D>, Stand: 28.08.2013.

Broccias, Cristiano. 2012. The syntax-lexicon continuum. In: Terttu Nevalainen \& Elizabeth Closs Traugott (Hg.). The Oxford Handbook of the History of English. Oxford: Oxford University Press, 735-747.

Busse, Dietrich. 2012. Frame-Semantik. Ein Kompendium. Berlin, Boston: De Gruyter.

Croft, William \& David A. Cruse. 2004. Cognitive Linguistics. Cambridge: Cambridge University Press.

Croft, William. 2001. Radical Construction Grammar. Syntactic Theory in Typological Perspective. Oxford: Oxford University Press.

Croft, William. 2013. Radical Construction Grammar. In: Thomas Hoffmann \& Graeme Trousdale (Hg.). The Oxford Handbook of Construction Grammar. Oxford: Oxford University Press.

Dowty, David R. 1991. Thematic Proto-Roles and Argument Selection. In: Language 67, 547-619.

Dudenredaktion (Hg.). 2009. Grammatik der deutschen Gegenwartssprache (Duden Band 4). 8., überarbeitete Aufl. Mannheim u. a.: Dudenverlag.

Eisenberg, Peter. 2006 I. Grundriss der deutschen Grammatik. Das Wort. 3., durchgesehene Aufl. Stuttgart, Weimar: Metzler.

Eisenberg, Peter. 2006 II. Grundriss der deutschen Grammatik. Der Sat\%. 3., durchgesehene Aufl. Stuttgart, Weimar: Metzler.

Eisenberg, Peter. 2011. Kategorienhierarchie und verbales Paradigma. Die analytischen Formen im Passiv, Perfekt, Konjunktiv. In: Oddleif Leirbukt (Hg.). Tempus/Temporalität und Modus/Modalität im Sprachenvergleich. 2., unveränderte Aufl. Tübingen: Stauffenburg, 1-24.

Eroms, Hans-Werner. 2000. Syntax der deutschen Sprache. Berlin, New York: De Gruyter.

Evans, Vyvyan \& Melanie Green. 2006. Cognitive Linguistics. An Introduction. Edinburgh: Edinburgh University Press.

Felfe, Marc. 2012. Das System der Partikelverben mit „an“. Eine konstruktionsgrammatische Untersuchung. Berlin, Boston: De Gruyter.

Fillmore, Charles J. 1968. The Case for Case. In: Emmon Bach \& Robert T. Harms (Hg.). Universals in Linguistic Theory. New York: Holt, Rinehart and Winston, 1-88.

Fillmore, Charles J. 1988. The Mechanisms of ,Construction Grammar'. In: Berkeley Linguistic Society 14, 35-55.

Fillmore, Charles J. 1989. Grammatical construction theory and the familiar dichotomies. In: Rainer Dietrich \& Carl F. Graumann (Hg.). Language processing in social context. Amsterdam: Elsevier Science, 17-38.

Goldberg, Adele E. 1995. Constructions: A Construction Grammar Approach to Argument Structure. Chicago u. a.: The University of Chicago Press. 
Goldberg, Adele E. 2003. Constructions: a new theoretical approach to language. In: Trends in Cognitive Sciences 7/5, 219-224.

Goldberg, Adele E. 2006. Constructions at Work. The Nature of Generalization in Language. Oxford: Oxford University Press.

Hilpert, Martin. 2013. Constructional Change in English. Developments in Allomorphy, Word Formation, and Syntax. Cambridge: Cambridge University Press.

Knobloch, Clemens. 2005. Oberfläche: metapragmatisch - zum Erwerb modalisierender Sprachzeichen. Siegen: Siegener Institut für Sprachen im Beruf.

Köller, Wilhelm. 1997. Funktionaler Grammatikunterricht: Tempus, Genus, Modus: wozu wurde das erfunden? Baltmannsweiler: Schneider-Hohengehren.

Lakoff, George. 1977. Linguistic gestalts. In: Papers from the 13th Regional Meeting of the Chicago Linguistic Society, 236-287.

Lakoff, George. 1987. Women, Fire, and Dangerous Things. Chicago: University Press of Chicago.

Langacker, Ronald W. 1982. Space Grammar, Analysability, and the English Passive. In: Language 58, 22-80.

Langacker, Ronald W. 1987. Foundations of Cognitive Grammar, Vol. 1, Theoretical Prerequisites. Stanford: Stanford University Press.

Langacker, Ronald W. 1988. An overview of cognitive grammar. In: Brygida RudzkaOstyn (Hg.). Topics in Cognitive Linguistics. Amsterdam, Philadelphia: Benjamins, 3-48.

Langacker, Ronald W. 1991. Foundations of Cognitive Grammar, Vol. 2, Descriptive Application. Stanford: Stanford University Press.

Langacker, Ronald W. 2005. Construction grammars: Cognitive radical, and less so. In: Francisco J. Ruiz de Mendoza Ibanez \& Sandra Pena Cervel (Hg.). Cognitive linguistics: Internal dynamics and interdisciplinary interaction. Berlin, New York: De Gruyter, 101-159.

Langacker, Ronald W. 2009. Cognitive (Construction) Grammar. In: Cognitive Linguitics 20/1, 167-176.

Lasch, Alexander. Im Druck. Rezension zu Marc Felfe. 2012. Das System der Partikelverben mit „an“. Eine konstruktionsgrammatische Untersuchung. Berlin, Boston: De Gruyter. In: Zeitschrift für Dialektologie und Linguistik.

Lenz, Alexandra N. 2013. Vom kriegen und bekommen. Kognitiv-semantische, variationslinguistische und sprachgeschichtliche Perspektiven. Berlin, New York: De Gruyter.

Maienborn, Claudia. 2003. Die logische Form von Kopula-Sätzen. Berlin: Akademie.

Michaelis, Laura A. 2003. Headless constructions and coercion by construction. In: Elaine Francis \& Michaelis, Laura A. (Hg.). Mismatch: Form-function incongruity and the architecture of grammar. Stanford: CSLI Publications, 259-310.

Petrova, Svetlana. 2008. Zur Interaktion von Tempus und Modus. Studien zur Entwicklungsgeschichte des Konjunktivs im Deutschen. Heidelberg: Winter.

Polenz, Peter von. 2008. Deutsche Satzsemantik. Grundbegriffe des Zwischen-den-Zeilen-Lesens. 3., unveränderte Aufl. der Ausgabe von 1985. Berlin, New York: De Gruyter.

Primus, Beatrice. 2012. Semantische Rollen. Heidelberg: Winter.

Smirnova, Elena \& Tanja Mortelmans. 2010. Funktionale Grammatik. Konzepte und Theorien. Berlin, New York: De Gruyter.

Stefanowitsch, Anatol \& Stefan Th. Gries. 2003. Collostructions: investigating the interaction between words and constructions. In: International Journal of Corpus Linguistics 8/2, 209-243.

Stefanowitsch, Anatol. 2009. Bedeutung und Gebrauch in der Konstruktionsgrammatik. Wie kompositionell sind modale Infinitive im Deutschen? In: Zeitschrift für Germanistische Linguistik 37/3, 565-592. 
Stefanowitsch, Anatol. 2011. Keine Grammatik ohne Konstruktionen: ein logischökonomisches Argument für die Konstruktionsgrammatik. In: Stefan Engelberg, Anke Holler \& Kristel Proost (Hg.). Sprachliches Wissen zwischen Lexikon und Grammatik. Berlin, New York: De Gruyter, 181-210.

Welke, Klaus. 2005. Tempus im Deutschen. Rekonstruktion eines semantischen Systems. Berlin, New York: De Gruyter.

Ziem, Alexander \& Alexander Lasch. 2013. Konstruktionsgrammatik. Konzepte und Grundlagen gebrauchsbasierter Ansätze. Berlin, Boston: De Gruyter. 
